\title{
A Robust Maximin Approach for MIMO Communications With Imperfect Channel State Information Based on Convex Optimization
}

\author{
Antonio Pascual-Iserte, Student Member, IEEE, Daniel Pérez Palomar, Member, IEEE, \\ Ana I. Pérez-Neira, Senior Member, IEEE, and Miguel Ángel Lagunas, Fellow, IEEE
}

\begin{abstract}
This paper considers a wireless communication system with multiple transmit and receive antennas, i.e., a multiple-input-multiple-output (MIMO) channel. The objective is to design the transmitter according to an imperfect channel estimate, where the errors are explicitly taken into account to obtain a robust design under the maximin or worst case philosophy. The robust transmission scheme is composed of an orthogonal space-time block code (OSTBC), whose outputs are transmitted through the eigenmodes of the channel estimate with an appropriate power allocation among them. At the receiver, the signal is detected assuming a perfect channel knowledge. The optimization problem corresponding to the design of the power allocation among the estimated eigenmodes, whose goal is the maximization of the signal-to-noise ratio (SNR), is transformed to a simple convex problem that can be easily solved. Different sources of errors are considered in the channel estimate, such as the Gaussian noise from the estimation process and the errors from the quantization of the channel estimate, among others. For the case of Gaussian noise, the robust power allocation admits a closed-form expression. Finally, the benefits of the proposed design are evaluated and compared with the pure OSTBC and nonrobust approaches.
\end{abstract}

Index Terms-Antenna arrays, beamforming, convex optimization theory, maximum optimization problems, multiple-input multiple-output (MIMO) systems, saddle point, space-time coding, worst-case robust designs.

\section{INTRODUCTION}

$\mathbf{M}$ ULTI-ANTENNA multiple-input-multiple-output (MIMO) channels have become a popular means to increase the spectral efficiency and the quality of wireless

Manuscript received September 27, 2004; revised March 16, 2005 This work was partially supported by the Spanish Government under projects TIC2002-04594-C02-01 (GIRAFA, jointly financed by FEDER) and FIT-070000-2003-257 (MEDEA+ A111 MARQUIS); by the European Commission under projects WIDENS (Contract FP6-507872) and IST-2002-2.3.1.4 (NEWCOM); and by the Fulbright Program and the Spanish Ministry of Education and Science. The associate editor coordinating the review of this manuscript and approving it for publication was Dr. Markus Rupp.

A. Pascual-Iserte and A. I. Pérez-Neira are with the Department of Signal Theory and Communications, Technical University of Catalonia (UPC), 08034 Barcelona, Spain (e-mail: tonip@gps.tsc.upc.es; anuska@gps.tsc.upc.es).

D. Pérez Palomar is with the Department of Electrical Engineering, Princeton University, Princeton, NJ 08544 USA (e-mail: danielp@princeton.edu).

M. Á. Lagunas is with the Telecommunications Technological Center of Catalonia (CTTC), 08034 Barcelona, Spain, and also with the Department of Signal Theory and Communications, Technical University of Catalonia (UPC), 08034 Barcelona, Spain (e-mail: m.a.lagunas@cttc.es).

Digital Object Identifier 10.1109/TSP.2005.861084 communications by means of spatial diversity at both sides of the link. The design of the communication system depends on the quantity and the quality of the channel state information (CSI) available at both the transmitter and the receiver. In case that the transmitter does not have any information about the channel, then space-time coding techniques should be applied [1]-[5]. The optimum exploitation of the benefits provided by MIMO systems can be achieved when a perfect CSI is available at both the transmitter and the receiver. In such a case, the optimum solution consists in the joint design of the transmitter and the receiver taking into account the channel response and the noise plus interference profile. In some works such as [6] and [7], and references therein, the design is done according to several performance criteria and using the tools and the potential provided by convex optimization theory [8].

In a realistic scenario, however, the channel knowledge is generally imperfect. In such a situation, the design should take into account explicitly the errors in the channel estimate, leading to robust designs less sensitive to these errors. The first applications of robust designs were not for wireless communications, but for control theory (see [9] and [10] and references therein). Indeed, the concepts of signal state space and MIMO were originally used in that area. Afterwards, all these techniques and concepts were extended to other fields due to their potential benefits. Some works such as [11]-[14] analyzed the performance degradation of several nonrobust solutions for multi-antenna systems, in which the errors in the CSI were considered negligible in the design process. The conclusion was that this degradation increases rapidly with the error level and, hence, robust designs are needed.

Regarding the CSI in a communication system, the receiver usually estimates the channel using a training sequence (pilot symbols). At the transmitter, the CSI can be obtained through a feedback channel or from previous received signals, exploiting the channel reciprocity in time division duplexing (TDD) (see [15] for an overview of different channel estimation strategies). Different sources of errors can be identified depending on the CSI acquisition method. In case of exploiting the channel reciprocity, the Gaussian noise from the estimation and the outdated estimate due to the channel variability have to be considered. If a feedback channel is used, additional effects arise, such as the quantization of the estimate and the errors in the communication through the feedback channel. 
The robust techniques can be classified into Bayesian (or stochastic) and maximin (or worst case) approaches, depending on the way the errors in the CSI are modeled [8], [16]. Bayesian techniques optimize a stochastic measure of the system performance, such as the mean or the outage values, assuming that the statistics of the error are known. On the other hand, maximin techniques consider that the error belongs to a predefined uncertainty region (with no inherent statistical assumption) and the final objective is the optimization of the worst system performance for any error in this region.

The Bayesian philosophy has been applied to systems with multi-antenna transmitters and single-antenna receivers in works such as [17]-[19] to optimize the signal-to-noise ratio (SNR), the bit error rate (BER), the mean square error (MSE), or the mutual information. The more general case of MIMO channels was considered in [20]-[22], and references therein. In some of these works, the transmitter was composed of an orthogonal space-time block code (OSTBC) and a beamforming stage, which was designed, for example, to minimize an upper bound of the BER. Two more examples are provided in [23] and [24], in which a statistical approach is taken to design a transmitter combining an Alamouti's code [1], a power allocation, and two beamformers to minimize an upper bound of the BER according to the knowledge of the channel correlation matrix and the channel mean, respectively. An overview of the analysis of the capacity of a MIMO system from an information theory point of view is given in [25]. There, a statistical approach is taken assuming that either the actual channel response or only its statistics are known at the transmitter and/or the receiver.

Regarding the maximin philosophy, [26] and [27] provide a general insight using a game theoretic formulation [28] and describing several applications in signal processing. This approach has been recently used in the classical problem of designing a receive beamformer under mismatches in the presumed model, as in [29], where the errors were assumed to be in the estimated steering vector and to belong to a spherical uncertainty region. This was afterwards generalized in [30] to embrace uncertainties both in the array response and the covariance matrix. The classical Capon's beamformer [31] was extended to its robust version in [32]-[34] taking generic uncertainty regions and different formulations. In some of these examples, the robustness was obtained by minimizing the output power of the beamformer while guaranteeing a minimum gain for any direction modeled by the uncertainty region. A linear receiver for a MIMO channel was derived in [35] to minimize the MSE, whereas in [36], the receivers for a multiuser scenario using OSTBC at the transmitters were obtained using a similar philosophy to that in [29]. A maximin design of a transmitter in a MIMO channel was proposed in [37] for the concrete case of spherical uncertainty regions. Finally, several applications of this robust approach to multiuser systems with multi-antenna base stations can also be found in [15], [38], [39].

In this paper, the objective is to obtain a robust maximin design of a communication system, where the channel estimate at the transmitter is imperfect. A MIMO channel is assumed, generalizing and improving other previous maximin designs, that considered, for example, multiple antennas only at one side of the system. The robust design problem is first formulated and, afterwards, is simplified to a convex optimization problem, for which global solutions can always be found, either analytically or numerically with a polynomial complexity [8]. Thanks to this transformation, the efficient software packages available for convex problems can be directly applied, obtaining the robust design (see [8] and [40] for a description of different techniques for solving convex problems). In some cases of practical interest, the simplified problem is quadratic and a simple closed-form solution can be found (see [8]).

The paper is organized as follows. In Section II, the system model is presented and the problem is formulated. The solution to the maximin problem is given in Section III based on convex optimization. Different uncertainty regions for the error in the channel estimate are described in Section IV, providing also a closed-form solution for the case of spherical uncertainty regions. An application of the robust design to adaptive modulation is presented in Section V. Finally, some simulations results and conclusions are provided in Sections VI and VII.

\section{System Model and Problem Statement}

Consider the transmission through a multi-antenna flat fading wireless MIMO channel with $n_{T}$ transmit and $n_{R}$ receive antennas. The $(i, j)$ th component of the channel matrix $\mathbf{H} \in \mathbb{C}^{n_{R} \times n_{T}}$ represents the channel gain between the $j$ th transmit and the $i$ th receive antenna. At the receiver, it is assumed that there is additive white Gaussian noise (AWGN) with power $\sigma_{n}^{2}$.

The objective in this work is to obtain a maximin robust design of the transmitter for an imperfect channel estimate $\hat{\mathbf{H}} \in$ $\mathbb{C}^{n_{R} \times n_{T}}$, which is modeled according to

$$
\mathbf{H}=\hat{\mathbf{H}}+\boldsymbol{\Delta}
$$

where $\Delta \in \mathbb{C}^{n_{R} \times n_{T}}$ is the error in the estimate. The CSI at the receiver is assumed to be perfect.

Consider, for illustrative purposes, that one symbol has to be transmitted at one time instant. In case that a perfect CSI is available, it has been shown in [6], [11] that the optimum solution maximizing the SNR is based on single beamforming, consisting in the transmission through the eigenvector of $\mathbf{H}^{H} \mathbf{H}$ associated to the maximum eigenvalue (the superscript $H$ stands for conjugate transpose). In case that the channel knowledge is imperfect, transmitting through the maximum eigenmode of $\hat{\mathbf{H}}^{H} \hat{\mathbf{H}}$ constitutes the nonrobust or naive solution, which may be quite sensitive to errors. Therefore, a robust design is expected to use more eigenmodes than the maximum one. See [37], for example, for a robust design using more estimated eigenmodes than the maximum one. Also in [41], a transmitter is designed according to different degrees of channel knowledge, showing that in some situations, the capacity achieving solution may need to use multiple beamforming.

The design of the transmitter will be based on a linear processing or beamforming scheme, whereas at the receiver, a maximum likelihood (ML) detector will be employed assuming a perfect channel knowledge. The proposed architecture for the robust transmitter is composed of an OSTBC block, whose outputs are transmitted through all the eigenmodes of $\hat{\mathbf{H}}^{H} \hat{\mathbf{H}}$ using an adequate power distribution among them, as opposed to the nonrobust design, which uses only the maximum eigenmode. 


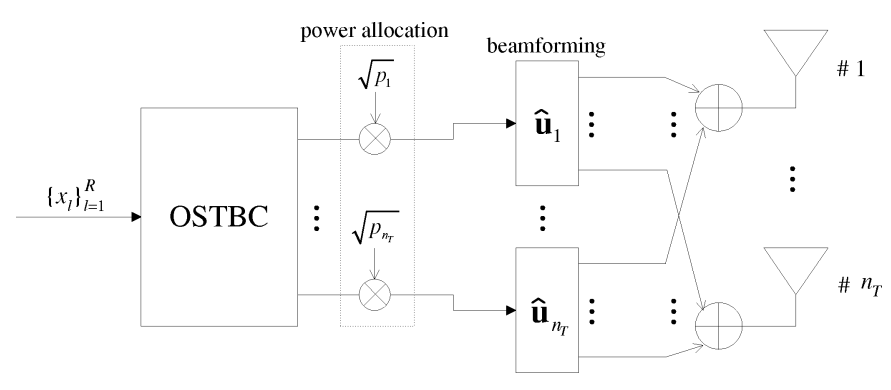

Fig. 1. General architecture for the transmitter based on the concatenation of an OSTBC block, a power allocation, and multiple beamforming.

The proposed architecture is shown in Fig. 1, where the OSTBC, the power allocation, and the beamforming stages are explicitly shown (similar transmitter architectures have been proposed in other works, such as [20], [22]-[24]).

Consider that an OSTBC block is used, such that $R$ independent complex symbols are transmitted simultaneously over $T$ periods of time, i.e., the code rate is $R / T$. According to this, the transmitted signal can be formulated as follows (similarly to linear dispersion codes [42] and OSTBC [4], [5]):

$$
\mathbf{S}=\hat{\mathbf{U}} \operatorname{diag}\left(\left\{\sqrt{p_{i}}\right\}\right) \sum_{l=1}^{R}\left(\mathbf{T}_{l}^{(r)} x_{l}^{(r)}+j \mathbf{T}_{l}^{(i)} x_{l}^{(i)}\right) \in \mathbb{C}^{n_{T} \times T}
$$

where each of the $n_{T}$ rows of $\mathbf{S}$ corresponds to the $T$ signal samples that are transmitted through each antenna, $x_{l}^{(r)}$ and $x_{l}^{(i)}$ are the real and imaginary parts of the complex symbol $x_{l}$, which is assumed to have a normalized energy $\mathbb{E}\left[\left|x_{l}\right|^{2}\right]=1$, and $\mathbf{T}_{l}^{(r)}$ and $\mathbf{T}_{l}^{(i)} \in \mathbb{C}^{n_{T} \times T}$ are the matrices belonging to the Hurwitz-Radon family of matrices used in OSTBC (see [4] and [5]). The unitary matrix $\hat{\mathbf{U}}=\left[\hat{\mathbf{u}}_{1} \cdots \hat{\mathbf{u}}_{n_{T}}\right] \in \mathbb{C}^{n_{T} \times n_{T}}$ contains the $n_{T}$ eigenvectors of $\hat{\mathbf{H}}^{H} \hat{\mathbf{H}}$ with eigenvalues $\left\{\hat{\lambda}_{i}\right\}$ sorted in decreasing order, $p_{i}$ is the power allocated to the transmission through the $i$ th estimated eigenmode, and $\operatorname{diag}\left(\left\{\sqrt{p_{i}}\right\}\right)$ is a diagonal matrix whose elements are $\left\{\sqrt{p_{i}}\right\}$. According to this signal model, the ML detector reduces to a set of linear operations, as happens with OSTBC.

The design objective is to calculate the optimum power allocation strategy $\left\{p_{i}\right\}$ subject to a transmit power constraint under an adequate performance criterion. If the transmit power budget is $P_{0}$, the power constraint can be expressed in terms of the factors $\left\{p_{i}\right\}$ as

$$
\frac{1}{R} \mathbb{E}\left[\|\mathbf{S}\|_{F}^{2}\right]=\sum_{i=1}^{n_{T}} p_{i} \leq P_{0}, \quad p_{i} \geq 0
$$

where $\|\mathbf{S}\|_{F}=\sqrt{\operatorname{Tr}\left(\mathbf{S}^{H} \mathbf{S}\right)}$ represents the Frobenius norm of matrix $\mathbf{S}$ and $\operatorname{Tr}(\cdot)$ is the trace operator. Note that the set of feasible power distributions is convex in $\left\{p_{i}\right\}$, since the constraints detailed in (3) are linear and, hence, convex [8]. Note also that, according to this notation, the nonrobust design corresponds to $p_{1}=P_{0}, p_{i}=0, i=2, \ldots, n_{T}$, i.e., only the maximum eigenmode is used for transmission [11]. Note that, regardless of the CSI, the symbol rate is always equal to the rate of the OSTBC
$R / T$, which means that the multiple beamforming architecture is used to provide robustness, but not for increasing the transmission rate of the code.

For the considered system (2) using OSTBC with ML detection, the performance can be measured by the SNR, which is equal for all the symbols and can be expressed as (see [4] and [5])

$$
\operatorname{SNR}=\frac{1}{\sigma_{n}^{2}} \operatorname{Tr}\left(\hat{\mathbf{U}}^{H} \mathbf{H}^{H} \mathbf{H} \hat{\mathbf{U}} \operatorname{diag}(\mathbf{p})\right)
$$

where $\mathbf{p}=\left[p_{1} \cdots p_{n_{T}}\right]^{T}$ (the superscript $T$ stands for transpose) and $\operatorname{diag}(\mathbf{p})$ is a diagonal matrix with elements $\left\{p_{i}\right\}$. Based on this, the performance function $f$ in this system can be defined as

$$
\begin{aligned}
f(\mathbf{p}, \boldsymbol{\Delta}) & =\operatorname{Tr}\left(\hat{\mathbf{U}}^{H} \mathbf{H}^{H} \mathbf{H} \hat{\mathbf{U}} \operatorname{diag}(\mathbf{p})\right) \\
& =\operatorname{Tr}\left(\hat{\mathbf{U}}^{H}(\hat{\mathbf{H}}+\boldsymbol{\Delta})^{H}(\hat{\mathbf{H}}+\boldsymbol{\Delta}) \hat{\mathbf{U}} \operatorname{diag}(\mathbf{p})\right)
\end{aligned}
$$

whose maximization with respect to $\mathbf{p}$ is the design objective and where the error model (1) has been used. Note that $f$ is linear and, therefore, concave in $\mathrm{p}$; and convex-quadratic in $\Delta .{ }^{1}$ The application of quasi-orthogonal space-time block codes [43] is not possible since they lead to nonlinear ML detectors whose performance cannot be directly measured by the SNR.

The maximin approach has been chosen to include robustness in the design of the power allocation. According to it, an uncertainty region $\mathcal{R}$ for the error in the estimate $\Delta$ has to be chosen, which, in the following, will be assumed to be a convex $\operatorname{set}^{2}[8]$ and to have a nonempty interior. This region models the imprecise knowledge of the channel and, therefore, the size of the region should be larger as the quality of the CSI decreases. The objective of the maximin design is to look for the power allocation $\mathbf{p}$ that optimizes the worst performance for any error in the uncertainty region, expressed as inf $\Delta \in \mathcal{R} f(\mathbf{p}, \boldsymbol{\Delta})$. Therefore, the robust approach can be formulated as [44]

$$
\begin{array}{cl}
\underset{\mathbf{p}}{\operatorname{maximize}} & \inf _{\boldsymbol{\Delta} \in \mathcal{R}} f(\mathbf{p}, \boldsymbol{\Delta}) \\
\text { subject to } & \mathbf{1}^{T} \mathbf{p} \leq P_{0} \\
& p_{i} \geq 0, \quad \forall i
\end{array}
$$

where $\mathbf{1}=[1 \cdots 1]^{T} \in \mathbb{R}^{n_{T} \times 1}$ is the all-one vector.

\section{Solving the Maximin Problem}

The direct way to solve the maximin problem is to obtain the minimization of $f$ analytically and then solve the outer maximization, either numerically or analytically. Such an approach, however, is difficult because it is not clear what is the minimizing $\Delta$ for a given $\mathrm{p}$ in closed-form.

\footnotetext{
${ }^{1}$ A function $f$ is convex if its domain $\operatorname{dom} f$ is a convex set, i.e., if $\mathbf{x}=$ $\theta \mathbf{x}_{1}+(1-\theta) \mathbf{x}_{2} \in \operatorname{dom} f, \forall \mathbf{x}_{1}, \mathbf{x}_{2} \in \operatorname{dom} f, \forall \theta \in[0,1]$, and the following is fulfilled: $f\left(\theta \mathbf{x}_{1}+(1-\theta) \mathbf{x}_{2}\right) \leq \theta f\left(\mathbf{x}_{1}\right)+(1-\theta) f\left(\mathbf{x}_{2}\right), \forall \mathbf{x}_{1}, \mathbf{x}_{2} \in$ $\operatorname{dom} f, \forall \theta \in[0,1]$. The function $f$ is concave if $-f$ is convex. See the chapter devoted to convex functions in [8] for a complete description of convex functions.

${ }^{2}$ The set $\mathcal{R}$ is convex if $\boldsymbol{\Delta}=\theta \boldsymbol{\Delta}_{1}+(1-\theta) \boldsymbol{\Delta}_{2} \in \mathcal{R}, \forall \boldsymbol{\Delta}_{1}, \boldsymbol{\Delta}_{2} \in \mathcal{R}$ and
} $\forall \theta \in[0,1]$. 
One can also consider the inner minimization numerically for a given $\mathbf{p}$

$$
\tilde{f}(\mathbf{p})=\inf _{\boldsymbol{\Delta} \in \mathcal{R}} f(\mathbf{p}, \boldsymbol{\Delta})
$$

and then solving again the outer maximization $\sup _{\mathbf{p}} \tilde{f}(\mathbf{p})$ numerically. Note that the inner minimization is a convex problem, since $f$ is convex in $\boldsymbol{\Delta}$ and the constraint set $\mathcal{R}$ is also convex. ${ }^{3}$ The outer maximization is also convex, since the constraint set for $\mathbf{p}$ is convex (the constraints in (3) are linear) and $\tilde{f}$ is concave [45]. This procedure allows to find the robust power allocation $\mathbf{p}^{\star}$, although it is computationally very costly. This is because each iteration in the outer maximization requires an evaluation of $\tilde{f}(\mathbf{p})$ (and possibly also of its gradient), which in turns requires solving the inner minimization numerically with as many iterations as needed to converge.

Other numerical methods can be used, such as the algorithm proposed in [46] to find saddle points of maximin problems based on a modified steepest descent over $p$ and $\Delta$ simultaneously. In [47], an alternative algorithm for the same problem is derived based on the interior point method.

In the following, a much more efficient and elegant way to solve the problem is shown, based on a transformation of the original maximin problem (6) into a simple convex optimization problem.

\section{A. Reformulating the Original Maximin Problem as a Simplified Convex Minimization Problem}

In this subsection, the original problem (6) is transformed into a simplified convex problem consisting of a single optimization stage, instead of the two stages defined originally (this equivalence was sketched in [45]). Thanks to this transformation, the powerful numerical algorithms available in software packages for solving convex problems can be applied to find the optimum solution to the design problem in a polynomial time, requiring much less computational effort than the algorithms previously mentioned. Some examples of these software packages are the optimization toolbox of MATLAB and SeDuMi [48]. This problem transformation is described in the following proposition:

Proposition 1: The original maximin problem (6) can be transformed into the following simplified convex optimization problem:

$$
\begin{array}{cl}
\underset{t, \Delta}{\operatorname{minimize}} & t \\
\text { subject to } & t \geq P_{0} \hat{\mathbf{u}}_{i}^{H}(\hat{\mathbf{H}}+\boldsymbol{\Delta})^{H}(\hat{\mathbf{H}}+\boldsymbol{\Delta}) \hat{\mathbf{u}}_{i}, \quad \forall i \\
& \boldsymbol{\Delta} \in \mathcal{R}
\end{array}
$$

where $t$ is a dummy variable and $\hat{\mathbf{u}}_{i}$ is the normalized eigenvector of $\hat{\mathbf{H}}^{H} \hat{\mathbf{H}}$ associated to the $i$ th eigenvalue (the eigenvalues are sorted in decreasing order). The optimum robust power allocation $\mathbf{p}^{\star}=\left[p_{1}^{\star} \cdots p_{n_{T}}^{\star}\right]^{T}$ is equal to the optimum dual variables $\left\{\gamma_{i}^{\star}\right\}$ associated to the inequalities $t \geq P_{0} \hat{\mathbf{u}}_{i}^{H}(\hat{\mathbf{H}}+\boldsymbol{\Delta})^{H}(\hat{\mathbf{H}}+\boldsymbol{\Delta}) \hat{\mathbf{u}}_{i}$ in (8) multiplied

\footnotetext{
${ }^{3} \mathrm{~A}$ constrained minimization problem is convex when both the objective function to be minimized and the constraint sets are convex (see [8]).
}

by the power budget $P_{0}$, i.e., $p_{i}^{\star}=P_{0} \gamma_{i}^{\star}$. Besides, the optimum primal variable $\boldsymbol{\Delta}^{\star}$ of (8) minimizes $f\left(\mathbf{p}^{\star}, \boldsymbol{\Delta}\right)$, i.e., $f^{\star} \triangleq f\left(\mathbf{p}^{\star}, \Delta^{\star}\right)=\tilde{f}\left(\mathbf{p}^{\star}\right)$.

Proof: Consider the original maximin problem

$$
\begin{array}{cl}
\underset{\mathbf{p}}{\operatorname{maximize}} & \inf _{\boldsymbol{\Delta} \in \mathcal{R}} \operatorname{Tr}\left(\hat{\mathbf{U}}^{H}(\hat{\mathbf{H}}+\boldsymbol{\Delta})^{H}(\hat{\mathbf{H}}+\boldsymbol{\Delta}) \hat{\mathbf{U}} \operatorname{diag}(\mathbf{p})\right) \\
\text { subject to } & \mathbf{1}^{T} \mathbf{p} \leq P_{0} \\
& p_{i} \geq 0, \quad \forall i
\end{array}
$$

where the optimum solution is attained when the transmit power constraint inequality is fulfilled with equality: $\mathbf{1}^{T} \mathbf{p}=P_{0}$. This problem can be rewritten in terms of the variables $\bar{p}_{i}=p_{i} / P_{0}$, so that the constraints are formulated as $\mathbf{1}^{T} \overline{\mathbf{p}}=1\left(\overline{\mathbf{p}}=\left[\bar{p}_{1} \ldots \bar{p}_{n_{T}}\right]^{T}\right)$ and $\bar{p}_{i} \geq 0, \forall i$. The problem is then

$$
\begin{array}{cl}
\underset{\overline{\mathbf{p}}}{\operatorname{maximize}} & \inf _{\boldsymbol{\Delta} \in \mathcal{R}} P_{0} \sum_{i=1}^{n_{T}} \bar{p}_{i} \hat{\mathbf{u}}_{i}^{H}(\hat{\mathbf{H}}+\boldsymbol{\Delta})^{H}(\hat{\mathbf{H}}+\boldsymbol{\Delta}) \hat{\mathbf{u}}_{i} \\
\text { subject to } & \mathbf{1}^{T} \overline{\mathbf{p}}=1 \\
& \bar{p}_{i} \geq 0, \quad \forall i .
\end{array}
$$

Let us include a dummy variable $t$, which, as will be shown in the following, is the same as the dummy variable $t$ in (8), obtaining

$$
\begin{array}{ll}
\underset{\overline{\mathbf{p}}}{\operatorname{maximize}} & \inf _{t, \boldsymbol{\Delta} \in \mathcal{R}} t+\sum_{i=1}^{n_{T}} \bar{p}_{i}\left(P_{0} \hat{\mathbf{u}}_{i}^{H}(\hat{\mathbf{H}}+\boldsymbol{\Delta})^{H}(\hat{\mathbf{H}}+\boldsymbol{\Delta}) \hat{\mathbf{u}}_{i}-t\right) \\
\text { subject to } & \mathbf{1}^{T} \overline{\mathbf{p}}=1 \\
& \bar{p}_{i} \geq 0, \quad \forall i
\end{array}
$$

It turns out that the constraint $\mathbf{1}^{T} \overline{\mathbf{p}}=1$ can now be removed since, if it is not satisfied, the inner minimization with respect to $t$ would be unbounded below (simply by looking at the term $\left.t\left(1-\sum_{i=1}^{n_{T}} \bar{p}_{i}\right)\right)$. Note that this constraint can be also derived as one of the Karush-Kuhn-Tucker (KKT) conditions [8] for the inner minimization in the previous problem. Hence, the original problem can be rewritten as

$$
\begin{array}{ll}
\underset{\overline{\mathbf{p}}}{\operatorname{maximize}} & \inf _{t, \boldsymbol{\Delta} \in \mathcal{R}} t+\sum_{i=1}^{n_{T}} \bar{p}_{i}\left(P_{0} \hat{\mathbf{u}}_{i}^{H}(\hat{\mathbf{H}}+\boldsymbol{\Delta})^{H}(\hat{\mathbf{H}}+\boldsymbol{\Delta}) \hat{\mathbf{u}}_{i}-t\right) \\
\text { subject to } & \bar{p}_{i} \geq 0, \quad \forall i
\end{array}
$$

This problem can be recognized as the maximization of the dual function (which in turn is defined as the minimization of the Lagrangian, as explained in [8]) associated to the problem

$$
\begin{array}{ll}
\underset{t, \Delta \in \mathcal{R}}{\operatorname{minimize}} & t \\
\text { subject to } & t \geq P_{0} \hat{\mathbf{u}}_{i}^{H}(\hat{\mathbf{H}}+\boldsymbol{\Delta})^{H}(\hat{\mathbf{H}}+\boldsymbol{\Delta}) \hat{\mathbf{u}}_{i}, \quad \forall i
\end{array}
$$

which is the same problem as (8) and, therefore, the dual variables or Lagrange multipliers $\gamma_{i}$ associated to the constraints $t \geq P_{0} \hat{\mathbf{u}}_{i}^{H}(\hat{\mathbf{H}}+\boldsymbol{\Delta})^{H}(\hat{\mathbf{H}}+\boldsymbol{\Delta}) \hat{\mathbf{u}}_{i}$ coincide with $\bar{p}_{i}$, i.e., $p_{i}=$ $P_{0} \gamma_{i}$, proving Proposition 1 . Note that the constraint $\boldsymbol{\Delta} \in \mathcal{R}$ is implicitly included in both problems (12) and (13) by defining the domain of the functions in the variable $\Delta$ as $\mathcal{R}$. 
From this proof, it can be seen that the fundamental reason why the relationship $p_{i}^{\star}=P_{0} \gamma_{i}^{\star}$ holds is that the original function $f$ is linear in the power distribution variables $\left\{p_{i}\right\}$ and, hence, they can be interpreted as the Lagrange multipliers.

In Appendix A, a completely different proof is given based on the application of the necessary and sufficient KKT conditions to the optimization problem (see [8] for a description of these conditions) and using the concepts of concave-convex functions and saddle points, also defined in the appendix.

Summarizing, the original maximin power allocation problem (6) can be solved by considering the simplified convex problem (8). The values of the optimum Lagrange multipliers for this problem provide the normalized power distribution to be applied among the estimated eigenmodes. Currently, there exist many software packages implementing very efficient numerical algorithms, such as the primal-dual interior point methods, that are able to solve convex optimization problems and give, not only the value of the optimum primal variables, i.e., $t^{\star}$ and $\boldsymbol{\Delta}^{\star}$ in problem (8), but also the optimum value of the dual variables, i.e., the Lagrange multipliers $\left\{\gamma_{i}^{\star}\right\}$. Consequently, by using these algorithms, the worst case error and the optimum robust power allocation can be calculated efficiently (see, for example, the function fmincon in the optimization toolbox of MATLAB). In addition, it can be shown that, for some concrete uncertainty regions $\mathcal{R}$, problem (8) simplifies to a quadratic problem and, even in some cases, a closed-form solution exists.

\section{B. An Application to Minimum Transmit Power With an Instantaneous Performance Constraint}

From the KKT conditions (37)-(40) in Appendix A for the reformulated convex problem (8), it can be shown that the optimal dual variables $\left\{\gamma_{i}^{\star}\right\}$ and the worst case error $\Delta^{\star}$ do not depend on the power budget $P_{0}$. Consequently, the optimum robust power allocation $\left(p_{i}^{\star}=P_{0} \gamma_{i}^{\star}\right)$ scales linearly with $P_{0}$, and also $f^{\star} \triangleq f\left(\mathbf{p}^{\star}, \boldsymbol{\Delta}^{\star}\right)$, which is given by

$$
\begin{aligned}
f^{\star} & \triangleq f\left(\mathbf{p}^{\star}, \boldsymbol{\Delta}^{\star}\right) \\
& =P_{0} \operatorname{Tr}\left(\hat{\mathbf{U}}^{H}\left(\hat{\mathbf{H}}+\boldsymbol{\Delta}^{\star}\right)^{H}\left(\hat{\mathbf{H}}+\boldsymbol{\Delta}^{\star}\right) \hat{\mathbf{U}} \operatorname{diag}\left(\left\{\gamma_{i}^{\star}\right\}\right)\right) .
\end{aligned}
$$

This result can be used to calculate the solution to the problem consisting in minimizing the transmit power, while still guaranteeing that the instantaneous performance, in terms of the SNR, is better than a minimum target $\mathrm{SNR}_{0}$ for any error in the uncertainty region. This problem is the complementary as that solved previously, in which the performance was optimized subject to a power constraint, although both problems are essentially equivalent. The solution to this new problem is also attained by taking the robust power allocation given by $\left\{\gamma_{i}^{\star}\right\}$, where the required transmit power is calculated as

$$
P_{0}^{\star}=\operatorname{SNR}_{0} \frac{\sigma_{n}^{2}}{\operatorname{Tr}\left(\hat{\mathbf{U}}^{H}\left(\hat{\mathbf{H}}+\boldsymbol{\Delta}^{\star}\right)^{H}\left(\hat{\mathbf{H}}+\boldsymbol{\Delta}^{\star}\right) \hat{\mathbf{U}} \operatorname{diag}\left(\left\{\gamma_{i}^{\star}\right\}\right)\right)} .
$$

The results in this paper can be straightforwardly extended to multicarrier systems assuming independent uncertainty regions for the channel estimate at each subcarrier and applying an adequate power allocation policy among the frequencies [11].

\section{CONVEX UnCERTAINTY REGIONS}

The definition of the uncertainty region $\mathcal{R}$ may impact importantly on the system performance. The size and the shape of this region should take into account the quality of the channel estimate and the imperfections that generate the error, linking the mathematical optimization problem and the physical phenomenon producing the error.

In the following, two sources of errors are identified and three different uncertainty regions, jointly with their sizes, are described. In all the cases, the proposed uncertainty regions are convex, as required to solve the optimization problem in Proposition 1. Afterwards, a more general list of possible uncertainty regions is given.

\section{A. Estimation Gaussian Noise}

A usual error in the channel estimate comes from the Gaussian noise, especially in TDD systems, where the transmitter can estimate the channel using the signals received in the reverse link, and use it as an estimate in the forward link, due to the channel reciprocity principle.

In this subsection, the objective is to derive the expression of an uncertainty region according to an unbiased estimate of the channel and taking into account that the error is Gaussian distributed. Let the unbiased channel estimate be formulated as $\tilde{\mathbf{H}}=\mathbf{H}+\mathbf{E}$, where $\mathbf{E}$ is the zero-mean estimation noise, independent from the actual channel realization. Note that a different notation is used for the unbiased channel estimate $\tilde{\mathbf{H}}$ and the estimation error $\mathbf{E}$ when compared to $\hat{\mathbf{H}}$ and $\boldsymbol{\Delta}$, as used in the previous sections. In the following, the relationship between $\hat{\mathbf{H}}, \tilde{\mathbf{H}}, \Delta$, and $\mathbf{E}$ is shown, and the corresponding uncertainty region for $\boldsymbol{\Delta}$ is obtained.

Let us define $\mathbf{h}=\operatorname{vec}(\mathbf{H})$ and $\mathbf{e}=\operatorname{vec}(\mathbf{E})$, where $\mathbf{h}$ and $\mathbf{e}$ are column vectors resulting from stacking the columns of $\mathbf{H}$ and $\mathbf{E}$, respectively, using the vec $(\cdot)$ operator. Frequently, $\mathbf{h}$ and $\mathbf{e}$ are assumed to be jointly Gaussian distributed with mean values and covariance matrices given by $\mathbf{m}_{h}$ and $\mathbf{C}_{h}$, and $\mathbf{0}$ and $\mathbf{C}_{e}$, for $\mathbf{h}$ and $\mathbf{e}$, respectively. According to this, the distribution of the actual channel conditioned to the unbiased channel estimate follows also a Gaussian distribution [49]:

$$
\begin{aligned}
\mathbf{h} \mid \tilde{\mathbf{h}} & \sim \mathcal{C N}\left(\mathbf{m}_{h \mid \tilde{h}}, \mathbf{C}_{h \mid \tilde{h}}\right), \\
& p_{h \mid \tilde{h}}(\mathbf{h} \mid \tilde{\mathbf{h}})=\frac{1}{\left|\pi \mathbf{C}_{h \mid \tilde{h}}\right|} e^{-\left(\mathbf{h}-\mathbf{m}_{h \mid \tilde{h}}\right)^{H} \mathbf{C}_{h \mid \tilde{h}}^{-1}\left(\mathbf{h}-\mathbf{m}_{h \mid \tilde{h}}\right)}
\end{aligned}
$$

where

$$
\begin{aligned}
\mathbf{m}_{h \mid \tilde{h}} & =\mathbf{m}_{h}+\mathbf{C}_{h}\left(\mathbf{C}_{h}+\mathbf{C}_{e}\right)^{-1}\left(\tilde{\mathbf{h}}-\mathbf{m}_{\tilde{h}}\right) \\
\mathbf{C}_{h \mid \tilde{h}} & =\left(\mathbf{C}_{h}^{-1}+\mathbf{C}_{e}^{-1}\right)^{-1} .
\end{aligned}
$$

Consequently, from (16) it is concluded that the actual channel $\mathbf{h}$ can be assumed to be in a region centered at $\mathbf{m}_{h \mid \tilde{h}}$, i.e., at the conditional mean of the actual channel, also known as the MMSE Bayesian channel estimate [49]. Based on this, $\hat{\mathbf{h}}=$ $\operatorname{vec}(\hat{\mathbf{H}})$ is defined as $\mathbf{m}_{h \mid \tilde{h}}$ and, therefore, the error $\boldsymbol{\delta}=\operatorname{vec}(\boldsymbol{\Delta})$ 


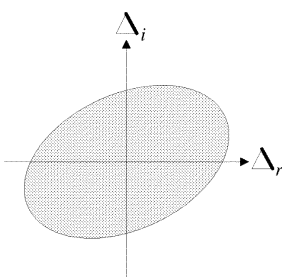

(a)

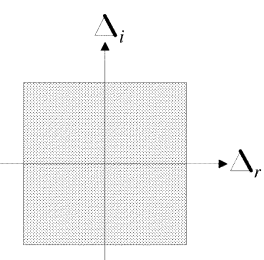

(b)

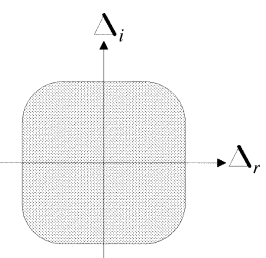

(c)
Fig. 2. Different uncertainty regions for the case of a scalar error $\Delta$, where $\Delta_{r}=\operatorname{Re}\{\Delta\}$ and $\Delta_{i}=\operatorname{Im}\{\Delta\}$ : (a) Estimation Gaussian noise, (b) quantization errors, and (c) combined estimation and quantization errors.

is equal to $\mathbf{h} \mathbf{-} \mathbf{m}_{h \mid \tilde{h}}$. According to these results, the uncertainty region for the error can be defined as the following ellipsoid (and, consequently, problem (8) is quadratic):

$$
\mathcal{R}=\left\{\boldsymbol{\Delta}: \boldsymbol{\delta}=\operatorname{vec}(\boldsymbol{\Delta}), \boldsymbol{\delta}^{H} \mathbf{C}_{h \mid \tilde{h}}^{-1} \boldsymbol{\delta} \leq r^{2}\right\}
$$

See Fig. 2(a) for an example of an ellipsoidal uncertainty region.

As the error $\boldsymbol{\delta}$ is Gaussian distributed, it will be inside the uncertainty region $\mathcal{R}$ with a certain probability $P_{\text {in }}$ lower than 1, i.e., $\operatorname{Prob}(\Delta \in \mathcal{R})=P_{\text {in }}<1$. This probability will be equal to the probability of providing the required QoS to the user (i.e., the probability of having a SNR higher than the target $\mathrm{SNR}_{0}$ or, equivalently, a BER lower than a maximum target $\mathrm{BER}_{0}$ ). The mathematical relationship between the size of the uncertainty region, measured by $r^{2}$, and $P_{\text {in }}$ is given by $r^{2}=\phi^{-1}\left(P_{\text {in }}\right)$, where $\phi$ is the cumulative density function (cdf) of the chi-square distribution with $2 n_{R} n_{T}$ degrees of freedom and normalized variance $1 / 2$ (this result can be easily obtained taking into account the statistical distribution (16) and that the vector $\mathbf{C}_{h \mid \tilde{h}}^{-1 / 2} \boldsymbol{\delta}$ is Gaussian distributed with zero-mean and identity covariance matrix).

For the concrete case where both the channel $\mathbf{H}$ and the error E matrices have independent identically distributed (i.i.d.) components with zero-mean and variances $\sigma_{h}^{2}$ and $\sigma_{e}^{2}$, respectively, the uncertainty region for the channel reduces to a sphere of radius $\sqrt{\epsilon}$ centered at the Bayesian channel estimate, obtaining

$$
\mathcal{R}=\left\{\boldsymbol{\Delta}:\|\boldsymbol{\Delta}\|_{F}^{2} \leq \epsilon, \epsilon=r^{2} \frac{\sigma_{h}^{2}}{1+\mathrm{SNR}_{\mathrm{est}}}\right\}
$$

where $\mathrm{SNR}_{\text {est }}=\sigma_{h}^{2} / \sigma_{e}^{2}$ is the received SNR during the transmission of the training sequence and $r^{2}$ is calculated as explained previously.

\section{B. Quantization Errors}

In frequency division duplexing (FDD) systems, the estimate of the channel at the transmitter has to be obtained through a feedback channel from the receiver to the transmitter. Since this feedback channel has a finite capacity, the channel response has to be quantized introducing an error in the CSI available at the transmitter. Assuming that the receiver has a perfect knowledge of the channel response $\mathbf{H}$, it can quantize uniformly the real and imaginary parts of all the components of $\mathbf{H}$ using a quantization step equal to $\Delta_{q}$, obtaining $\hat{\mathbf{H}}$ as a result. Taking this parameter, the quantization $\mathrm{SNR}$ is defined as $\mathrm{SNR}_{q}=6 \sigma_{h}^{2} / \Delta_{q}^{2}$, where $\sigma_{h}^{2}$ is the variance of each component of $\mathbf{H}$ assuming i.i.d. Gaussian components. Consequently, the uncertainty region for the channel can be defined as a hypercube centered at $\hat{\mathbf{H}}$ and, therefore, $\mathcal{R}$ is defined as follows (leading to a convex quadratic optimization problem):

$$
\mathcal{R}=\left\{\boldsymbol{\Delta}:\left|\operatorname{Re}\left\{[\boldsymbol{\Delta}]_{i j}\right\}\right| \leq \frac{\Delta_{q}}{2},\left|\operatorname{Im}\left\{[\boldsymbol{\Delta}]_{i j}\right\}\right| \leq \frac{\Delta_{q}}{2}\right\} .
$$

See Fig. 2(b) for an example of a cubic uncertainty region.

Usually, the assumed dynamic range for the quantization is equal to 6 times the standard deviation. If this approach is taken, the relationship between $\mathrm{SNR}_{q}$ and the number of bits $N_{b}$ to be fed back is $N_{b}=n_{T} n_{R} \log _{2}\left(3 \mathrm{SNR}_{q}\right)$. Obviously, as the capacity of the feedback channel increases, more bits can be used in the quantization and, therefore, the size of the uncertainty region can be reduced.

\section{Combined Estimation and Quantization Errors}

In a realistic scenario, the two effects considered previously, i.e., the Gaussian noise from the estimation process and the quantization errors, are expected to be combined. This can be modeled mathematically by defining an appropriate uncertainty region for the error, which can be expressed as

$$
\mathcal{R}=\left\{\begin{aligned}
\boldsymbol{\Delta}= & \boldsymbol{\Delta}_{1}+\boldsymbol{\Delta}_{2}:\left\|\boldsymbol{\Delta}_{1}\right\|_{F}^{2} \leq \epsilon, \\
& \left|\operatorname{Re}\left\{\left[\boldsymbol{\Delta}_{2}\right]_{i j}\right\}\right| \leq \frac{\Delta_{q}}{2},\left|\operatorname{Im}\left\{\left[\boldsymbol{\Delta}_{2}\right]_{i j}\right\}\right| \leq \frac{\Delta_{q}}{2}
\end{aligned}\right\}
$$

and is convex. See Fig. 2(c) for an example of this uncertainty region. Note that in this case, white Gaussian noise and uncorrelated MIMO channels have been considered, although the extension to ellipsoidal regions combined with quantization is straightforward. According to this region, the optimization problem (8) can be rewritten as the following convex quadratic problem:

$$
\begin{array}{cl}
\underset{t, \boldsymbol{\Delta}_{1}, \boldsymbol{\Delta}_{2}}{\operatorname{minimize}} & t \\
\text { subject to } \quad & t \geq \\
& P_{0} \hat{\mathbf{u}}_{i}^{H}\left(\hat{\mathbf{H}}+\boldsymbol{\Delta}_{1}+\boldsymbol{\Delta}_{2}\right)^{H} \\
& \times\left(\hat{\mathbf{H}}+\boldsymbol{\Delta}_{1}+\boldsymbol{\Delta}_{2}\right) \hat{\mathbf{u}}_{i}, \quad \forall i \\
& \operatorname{Tr}\left(\boldsymbol{\Delta}_{1}^{H} \boldsymbol{\Delta}_{1}\right) \leq \epsilon \\
& \left|\operatorname{Re}\left\{\left[\boldsymbol{\Delta}_{2}\right]_{i j}\right\}\right| \leq \frac{\Delta_{q}}{2},\left|\operatorname{Im}\left\{\left[\boldsymbol{\Delta}_{2}\right]_{i j}\right\}\right| \leq \frac{\Delta_{q}}{2}
\end{array}
$$

which comprises the previous uncertainty regions and the corresponding optimization problems as particular cases.

\section{Other Uncertainty Regions}

In addition to the previous uncertainty regions, there are many other possibilities and feedback strategies, whose error and imperfection models also lead to convex uncertainty regions. The regions described previously make sense when the whole channel matrix $\mathbf{H}$ is estimated or each element of $\mathbf{H}$ is independently quantized. In the case of SIMO channels, a possible approach is to represent the channel in terms of the modes of the channel correlation matrix, as in the Karhunen-Loeve transform (see [50] and references therein). Consider the channel $\mathbf{h}$, where this column vector represents the response of the SIMO channel with fixed covariance matrix $\mathbb{E}\left[\mathbf{h h}^{H}\right]=\mathbf{U D U}^{H}$. Given this eigen-decomposition, the actual channel response and the estimate can be coded taking the eigenvectors as the basis vectors for the representation, 
obtaining: $\alpha_{i}=\mathbf{u}_{i}^{H} \mathbf{h}$, so that $\mathbf{h}=\sum_{i} \alpha_{i} \mathbf{u}_{i}$ and $\hat{\mathbf{h}}=\sum_{i} \hat{\alpha}_{i} \mathbf{u}_{i}$ (the eigenvectors are assumed to be known both at the transmitter and the receiver, and the only parameters that have to be fed back are the coefficients $\alpha_{i}$ ).

In a general setup for a MIMO channel, this strategy could also be used and, therefore, the representation of the MIMO channel in terms of its coordinates $\left\{\alpha_{i}\right\}$ could be expressed as

$$
\mathbf{H}=\sum_{i} \alpha_{i} \mathbf{H}_{i}
$$

where the matrices $\left\{\mathbf{H}_{i}\right\}$, which are fixed and known at both sides of the system, form the basis for the expression of the channel estimate. The estimated/quantized channel available at the transmitter is

$$
\hat{\mathbf{H}}=\sum_{i} \hat{\alpha}_{i} \mathbf{H}_{i}
$$

where the error in the coefficients is represented by $\delta_{i}=\alpha_{i}-\hat{\alpha}_{i}$. If the set defined for the error vector $\boldsymbol{\delta}=\left[\delta_{1} \ldots \delta_{N}\right]^{T}$ is convex, then the uncertainty region for the error in the channel estimate $\boldsymbol{\Delta}=\mathbf{H}-\hat{\mathbf{H}}=\sum_{i} \delta_{i} \mathbf{H}_{i}$ is also convex, since the channel is expressed as a linear combination of the matrices of the basis multiplied by the coefficients $\left\{\alpha_{i}\right\}$ [8].

The imperfections and uncertainty regions described previously can also be adopted for the coordinates $\left\{\alpha_{i}\right\}$ as follows:

1) spherical/ellipsoidal regions for the coordinates-represented by $\sum_{i} w_{i}\left|\delta_{i}\right|^{2} \leq r^{2}$

2) quantization regions for the coordinates-represented by $\left|\operatorname{Re}\left\{\delta_{i}\right\}\right| \leq \Delta_{q} / 2,\left|\operatorname{Im}\left\{\delta_{i}\right\}\right| \leq \Delta_{q} / 2$

3) combination of 1) and 2).

Previously, the quantization has been assumed to be a scalar quantization; however, a vector quantization is usually preferred. Consider a space with $N$ points $\left\{\mathbf{H}_{i}\right\}$, each one representing the region given by $\mathbf{H}_{i}+\mathcal{R}_{i}$, i.e., if $\mathbf{H} \in \mathbf{H}_{i}+\mathcal{R}_{i}$, the $i$ th index corresponding to $\mathbf{H}_{i}$ is sent (the number of bits for the feedback is equal to $\log _{2}(N)$ ). Each region $\mathcal{R}_{i}$ is a polyhedron defined by the intersection of a finite number of half-spaces (see Fig. 3 for an example). The region is then as follows:

4) vector quantization- $\hat{\mathbf{H}}=\mathbf{H}_{i}$ and $\boldsymbol{\Delta} \in \mathcal{R}_{i}$, where $i$ is the received index; observe that in this case, the uncertainty regions depends on the channel estimate, as opposed to the previous cases where it was fixed.

See [51], and references therein, for a general overview of different vector quantization strategies with limited feedback.

\section{E. A Closed-Form Solution for Spherical Uncertainty Regions}

The general convex problem presented in (8) can be extremely simplified for the case of spherical uncertainty regions. In this subsection, a closed-form solution is given for this region, improving the results given in [44], in which the solution was not exact and an iterative method had to be applied. The solution provided in this subsection is similar to that obtained in the independent work [37] but using a completely different proof. There, a maximin approach has also been taken to find a solution to the problem of the maximization of the SNR

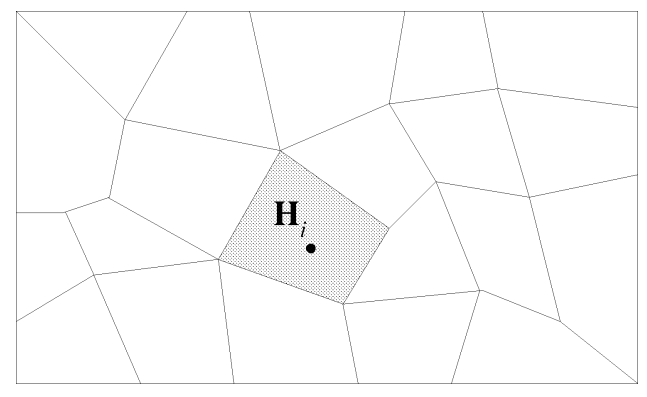

Fig. 3. Uncertainty regions resulting from the intersection of half-spaces in vector quantization. In this example, the channel is in the indicated region and the index $i$ is fed back to the transmitter.

assuming spherical uncertainty regions with a prefixed size, where no discussion is given on how to calculate the value of the radius of the region. That paper assumes a different transmitter architecture from the one presented in this paper, proposing the use of a spreading sequence at each antenna instead of an OSTBC and, therefore, leading to transmission rates much lower than those obtained by our proposal.

Proposition 2: Consider the maximin problem (6) and the uncertainty region $\mathcal{R}=\left\{\|\boldsymbol{\Delta}\|_{F}^{2} \leq \epsilon\right\}$. If $\|\hat{\mathbf{H}}\|_{F}^{2} \leq \epsilon$, then $\sup _{\mathbf{p}} \inf _{\boldsymbol{\Delta}} f(\mathbf{p}, \boldsymbol{\Delta})=0$ and no SNR can be guaranteed, i.e., no robust power allocation exists. Otherwise, the optimum robust power allocation is given by

$$
p_{i}^{\star}= \begin{cases}\mu\left(\sqrt{\hat{\lambda}_{i}}-\alpha\right), & 1 \leq i \leq i_{\max } \\ 0, & i>i_{\max }\end{cases}
$$

where $\hat{\lambda}_{i}$ is the $i$ th eigenvalue of $\hat{\mathbf{H}}^{H} \hat{\mathbf{H}}$ (with the eigenvalues sorted in decreasing order), $\mu$ is a normalization factor such that $\sum_{i=1}^{n_{T}} p_{i}^{\star}=P_{0}, i_{\max }$ is the maximum index such that the following inequality is fulfilled:

$$
\sum_{i=1}^{i_{\max }}\left(\sqrt{\hat{\lambda}_{i}}-\sqrt{\hat{\lambda}_{i_{\max }}}\right)^{2}<\epsilon
$$

and $\alpha$ is the minimum solution to the following second degree equation:

$$
i_{\max } \alpha^{2}-\left(2 \sum_{i=1}^{i_{\max }} \sqrt{\hat{\lambda}_{i}}\right) \alpha+\sum_{i=1}^{i_{\max }} \hat{\lambda}_{i}-\epsilon=0 .
$$

Proof: See Appendix B.

\section{Application To Adaptive Modulation WITH BER CONSTRAINTS}

The previous robust design can be combined with adaptive modulation strategies [52] to maximize the throughput subject to BER constraints, i.e., the objective is to maximize the transmission rate by employing high level modulations while still guaranteeing a minimum quality in terms of a maximum BER for any possible error in the uncertainty region.

Consider that the transmit power is bounded by $P_{0}^{\max }$ and let $\mathrm{BER}_{L}(\mathrm{SNR})$ be the function that relates the SNR with the BER for an $L$-QAM modulation. Obviously, given a certain SNR, the BER increases as the number of constellation points $L$ in the modulation also increases. Taking this into account, the 
proposed robust adaptive modulation is based on the following steps, in which all the symbols are assumed to be taken from the same signal constellation:

1) Define the desired QoS in terms of a maximum allowed $\mathrm{BER}_{0}$.

2) Given the channel estimate $\hat{\mathbf{H}}$ and the uncertainty region $\mathcal{R}$, calculate the robust normalized power allocation given by the optimum Lagrange multipliers $\left\{\gamma_{i}^{\star}\right\}$ and the worst case error given by the optimum primal variables $\Delta^{\star}$.

3) Calculate the guaranteed achievable SNR for any error in the uncertainty region similarly as in (15), as follows:

$$
\begin{aligned}
& \mathrm{SNR}_{\max }^{\max } \\
& \quad=\frac{P_{0}^{\max }}{\sigma_{n}^{2}} \operatorname{Tr}\left(\hat{\mathbf{U}}^{H}\left(\hat{\mathbf{H}}+\boldsymbol{\Delta}^{\star}\right)^{H}\left(\hat{\mathbf{H}}+\boldsymbol{\Delta}^{\star}\right) \hat{\mathbf{U}} \operatorname{diag}\left(\left\{\gamma_{i}^{\star}\right\}\right)\right) .
\end{aligned}
$$

4) Calculate the maximum number of constellation points $L^{\star}$ fulfilling $\mathrm{BER}_{L}\left(\mathrm{SNR}^{\max }\right) \leq \mathrm{BER}_{0}$. This can be trivially done by using a look-up table in which the values of the SNR required for each BER and signal constellation size are saved. If the previous constraint cannot be fulfilled for any value of $L$, set $L^{\star}=0$. In this case, no signal is transmitted since the QoS requirement cannot be satisfied for all the possible errors in the uncertainty region while still fulfilling the maximum transmit power constraint.

5) For the selected value $L^{\star}$, calculate the necessary instantaneous transmit power as in (15). Note that the transmit power required to fulfill the BER constraint with equality may be lower than the maximum available transmit power $P_{0}^{\max }$ since the number of constellation points $L$ is discrete.

Summarizing, this algorithm proposes a robust adaptive modulation technique, in which the throughput is maximized while a certain QoS can be guaranteed to the user given a channel estimate and a transmit power constraint.

\section{Simulations RESUlts}

In this section, several simulations results are presented to show the robustness capabilities of the already presented technique and compare its performance with other classical solutions, such as the nonrobust approach and the pure OSTBC approach. In all the simulations in this section, the OSTBC matrices given in [4] for complex symbols are used, attaining a symbol rate $R / T$, according to the notation used in (2), equal to 1 for $n_{T}=2,3 / 4$ for $n_{T}=3$ and $n_{T}=4$, and $1 / 2$ for any other number of transmit antennas. In the simulations, the optimum robust power allocation has been obtained simply using the function fmincon of the optimization toolbox of MATLAB, that provides simultaneously the optimum values of the primal and dual variables of problem (8).

As presented in Section III, the robust maximin technique distributes the available power among the estimated eigenmodes taking into account the errors in the channel estimate. If the channel estimate is perfect, the robust solution should be equal to the nonrobust beamforming, i.e., to the power allocation given by $p_{1}=P_{0}, p_{i}=0, i=2, \ldots, n_{T}$. When the uncertainty in the

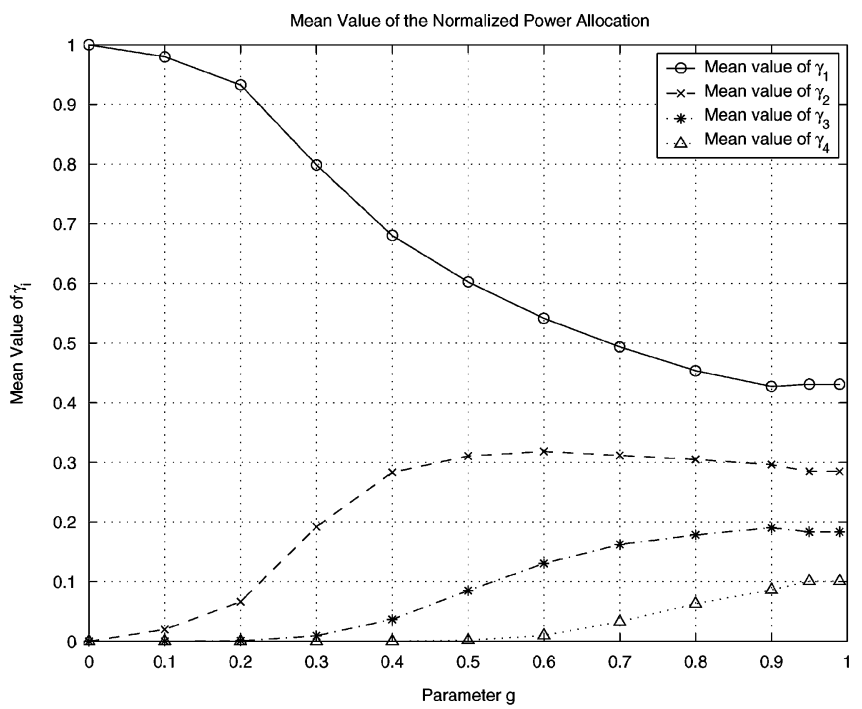

Fig. 4. Mean value of the power distribution for different sizes of the uncertainty region.

actual channel increases, the robust design tends to distribute the power in a more uniform way.

In the first simulations we analyze a system with 4 transmit and 6 receive antennas. We consider Gaussian noise in the channel estimate and spherical uncertainty regions with a radius equal to $\sqrt{\epsilon}=g\|\hat{\mathbf{H}}\|_{F}, 0 \leq g<1$. Note that for these uncertainty regions, $\mathbf{H}=\hat{\mathbf{H}}+\boldsymbol{\Delta} \neq \mathbf{0}, \forall \boldsymbol{\Delta} \in \mathcal{R}$. This condition has to hold since, otherwise, the saddle value, i.e., $f\left(\mathbf{p}^{\star}, \boldsymbol{\Delta}^{\star}\right)$, would be equal to 0 .

Since $n_{T}=4$, the total transmit power has to be distributed among the 4 estimated eigenvectors. In Fig. 4, the mean value of the normalized robust power allocation $\left\{\gamma_{i}^{\star}\right\}$ is shown as a function of $g$. As seen, for $g=0$ the power distribution corresponds to the nonrobust approach, as expected. As $g$ increases, the power allocation profile changes and tends to distribute the power in a more uniform way. Note that the pure OSTBC approach is equivalent to a uniform power allocation $p_{i}=1 / 4=$ $0.25,1 \leq i \leq 4$. As can be seen in Fig. 4 , this uniform distribution is not attained by the robust approach, even when $g \rightarrow 1$. In [5], it is shown that OSTBC is optimum in a robust maximin sense when the channel is completely unknown. This can be expressed mathematically as the following nonconvex uncertainty region for the actual channel: $\tilde{\mathcal{R}}=\left\{\mathbf{H}:\|\mathbf{H}\|_{F}^{2} \geq \rho\right\}$, where $\rho$ is a positive real value that avoids the channel $\mathbf{H}=\mathbf{0}$ to belong to the uncertainty region. Note that, when $g=1$, the spherical uncertainty region is different from that for which OSTBC is the robust maximin solution and, consequently, the robust power allocation policy for $g=1$ does not have to be necessarily uniform.

As stated in Subsection III-B, the maximin design can be used to guarantee a minimum target $\mathrm{SNR}_{0}$ with the minimum required transmit power for any error in the uncertainty region. In Fig. 5, the cdf of the minimum required transmit power is shown for $\mathrm{SNR}_{0}=10 \mathrm{~dB}$. This cdf is represented for $n_{T}=2$ and $n_{R}=2$ and for three different transmission techniques: the robust approach, the nonrobust classical beamforming, and a pure OSTBC strategy. The uncertainty regions that are considered are spherical with a radius equal to $\sqrt{\epsilon}=g\|\hat{\mathbf{H}}\|_{F}$ and 


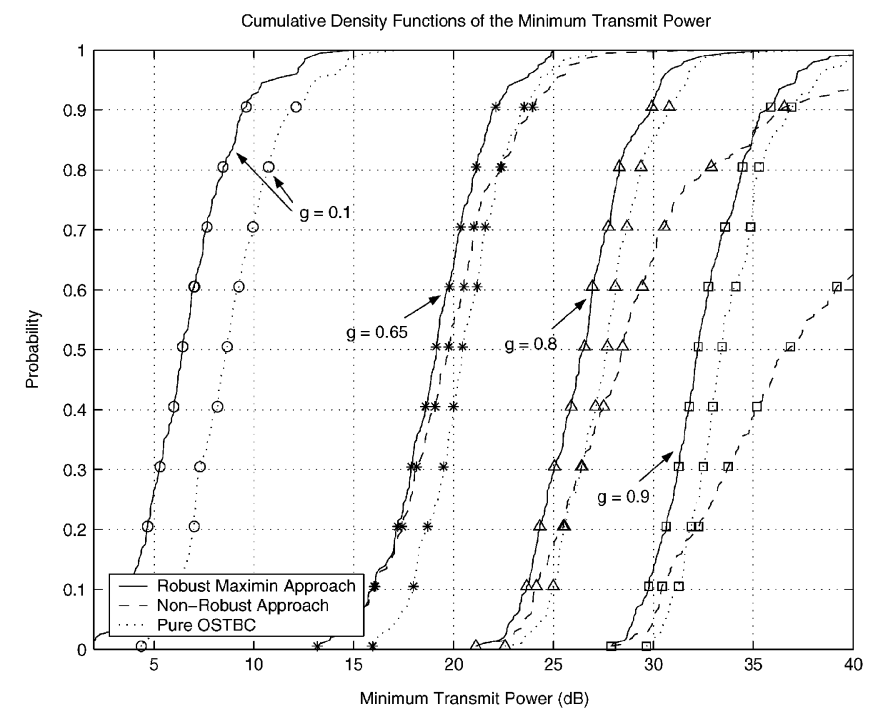

Fig. 5. Cumulative density functions of the minimum required transmit power assuming different sizes for the spherical uncertainty regions, and according to a target SNR equal to $\mathrm{SNR}_{0}=10 \mathrm{~dB}$.

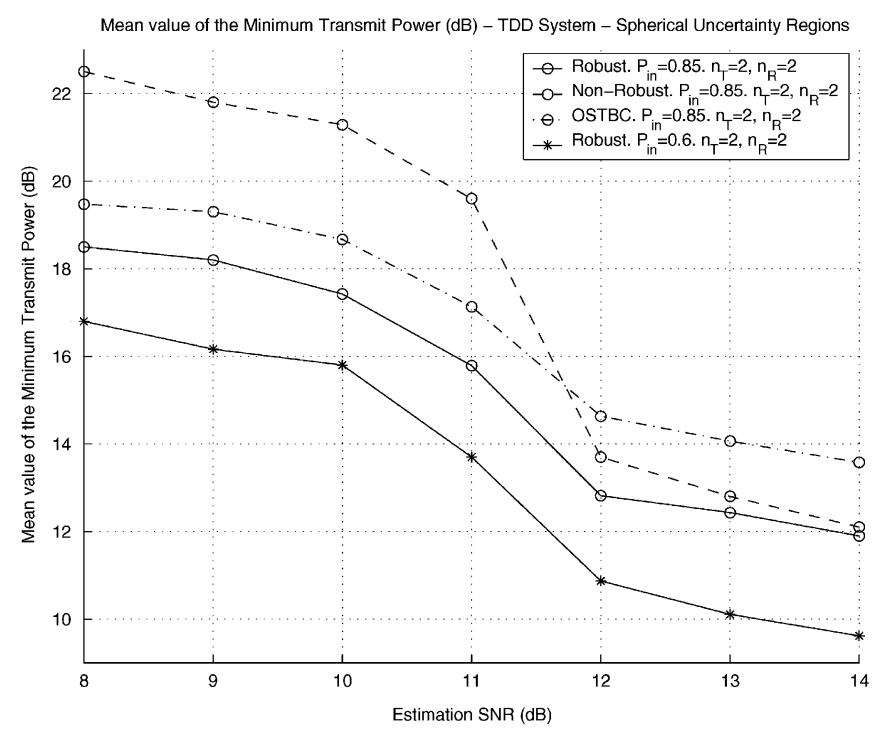

Fig. 6. Mean value of the minimum required transmit power in a TDD system (Gaussian noise, spherical uncertainty regions) according to a target SNR equal to $\mathrm{SNR}_{0}=10 \mathrm{~dB}$.

taking four different values for the parameter $g: 0.1,0.65,0.8$, and 0.9. As can be seen, for small uncertainty regions, both the robust and the nonrobust approaches have a similar performance and need less transmit power than OSTBC, as expected. When the size of the uncertainty region increases, the nonrobust approach increases the necessary transmit power to fulfill the QoS requirements. Note that, for an extreme case corresponding to big uncertainty regions, the nonrobust technique may need even more power than OSTBC. This means that in case that the CSI may have high errors, it is more convenient to use an OSTBC approach than to assume that the channel estimate is perfect, despite not being true. Note also that, in all cases, the robust solution is the technique requiring the least transmit power.

Figs. 6 and 7 show some results on the mean value of the minimum required transmit power to attain a $\mathrm{SNR}_{0}=10 \mathrm{~dB}$ when

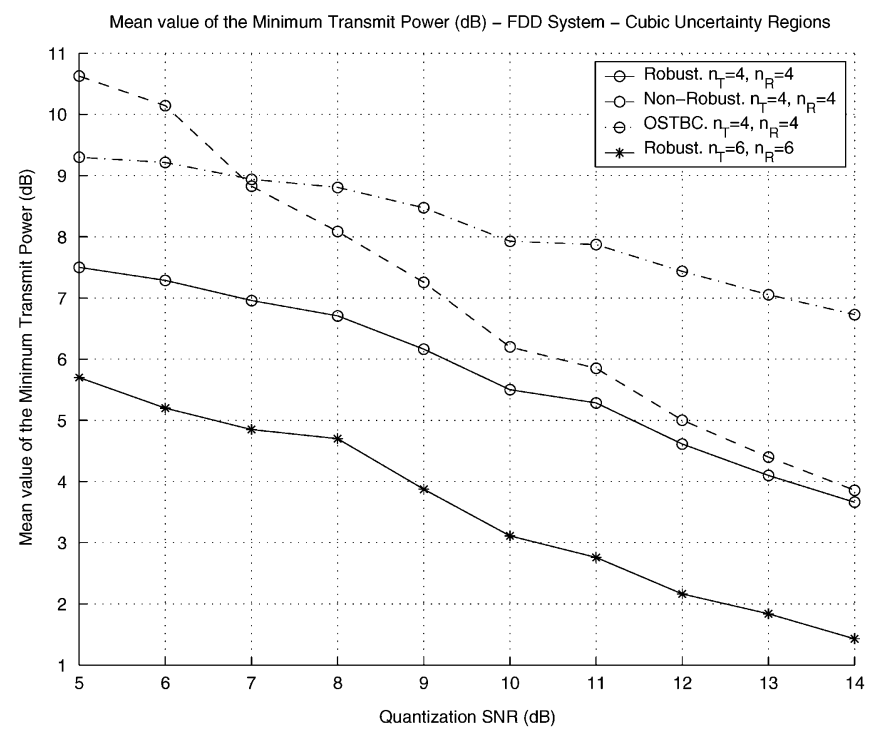

Fig. 7. Mean value of the minimum required transmit power in a FDD system (quantization errors, cubic uncertainty regions) according to a target SNR equal to $\mathrm{SNR}_{0}=10 \mathrm{~dB}$.

considering spherical (TDD systems) and cubic (FDD systems) uncertainty regions. The sizes of the regions are directly related to the estimation and quantization SNR, as described in Section IV. For the case of spherical uncertainty regions, two different QoS probabilities (as defined in Section IV-A) have been used: $P_{\text {in }}=0.85$ and $P_{\text {in }}=0.6$. The same conclusions can be obtained from the observation of both figures. If the estimation or quantization SNR is high, OSTBC needs more power than the nonrobust and the robust designs, since it does not exploit the channel knowledge available at the transmitter. As the estimation or quantization SNR decreases, all the techniques need more power to fulfill the instantaneous SNR requirements, since the size of the uncertainty region increases. Note that for all the cases, the technique requiring the least transmit power is the robust approach. Also, as previously pointed out, if the estimation or quantization SNR is low enough, the nonrobust solution needs more power than OSTBC, concluding that in case of having a very low quality channel estimate, it is not convenient to use it without taking into account explicitly the errors in the estimate, i.e., in a nonrobust way. In the case of spherical regions, increasing the probability $P_{\text {in }}$ of providing a QoS implies an increase of the minimum required transmit power, since the size of the uncertainty region also increases. From the figures it is also concluded that very important savings in terms of transmit power can be obtained when using the robust approach instead of the nonrobust beamforming. For example, for $n_{T}=2$ and $n_{R}=2$ and $\mathrm{SNR}_{\text {est }}=11 \mathrm{~dB}$, a saving of almost $4 \mathrm{~dB}$ can be obtained, whereas for $n_{T}=6$ and $n_{R}=6$ and $\mathrm{SNR}_{q}=5 \mathrm{~dB}$, a saving of $3 \mathrm{~dB}$ is achieved.

As explained at the beginning of this section, an instantaneous SNR can be guaranteed to the user only in case that $\mathbf{H}=\mathbf{0}$ does not belong to the uncertainty region for the actual channel. In Figs. 8 and 9 the service provision probability is shown, i.e., $\operatorname{Pr}(\mathbf{H}+\boldsymbol{\Delta} \neq \mathbf{0}, \forall \boldsymbol{\Delta} \in \mathcal{R})$, as a function of the QoS probability $P_{\text {in }}$ required by the user in the case of TDD, and the quantization SNR in the case of FDD. These results have been obtained 


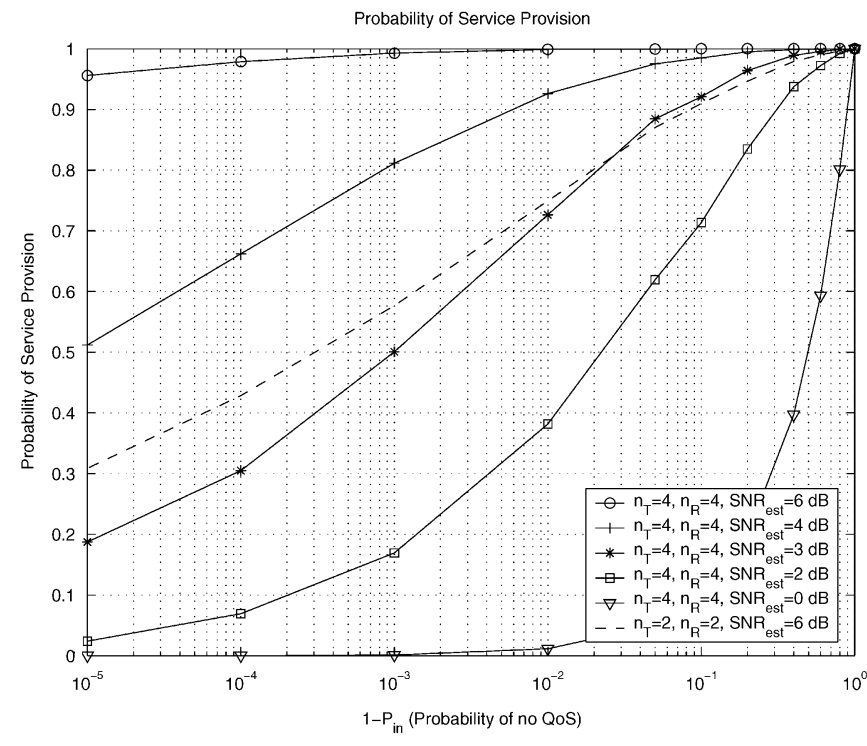

Fig. 8. Probability of service provision versus $1-P_{\text {in }}$, where $P_{\text {in }}$ is the probability of attaining the desired QoS assuming Gaussian noise and spherical uncertainty regions.

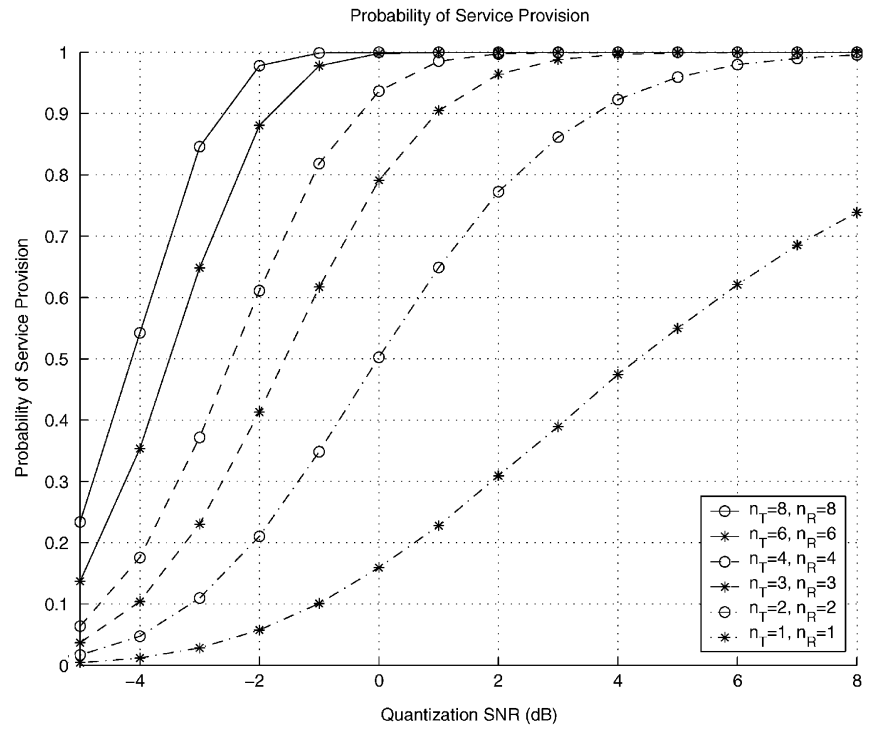

Fig. 9. Probability of service provision versus quantization SNR, assuming quantization errors and cubic uncertainty regions.

for different number of transmit and receive antennas. As a general conclusion, it can be observed that increasing the number of antennas and the quantization SNR implies an increase of the service provision probability, as expected. On the other hand, if the user demands for a higher QoS probability, the service provision probability decreases, since an increase of the required QoS implies an increase of the uncertainty region $\mathcal{R}$ and, therefore, it is not always possible to guarantee that QoS since the actual channel $\mathbf{H}=\mathbf{0}$ may be possible.

As explained in Section V, the throughput, i.e., the number of transmitted bits per channel use, can be maximized while guaranteeing a maximum BER for any possible error in the channel estimate by using the robust power allocation. Fig. 10 shows the throughput (averaged over many channel realizations) that can be achieved in order to guarantee a maximum BER equal to $10^{-3}$ for any possible error in the uncertainty region, which is

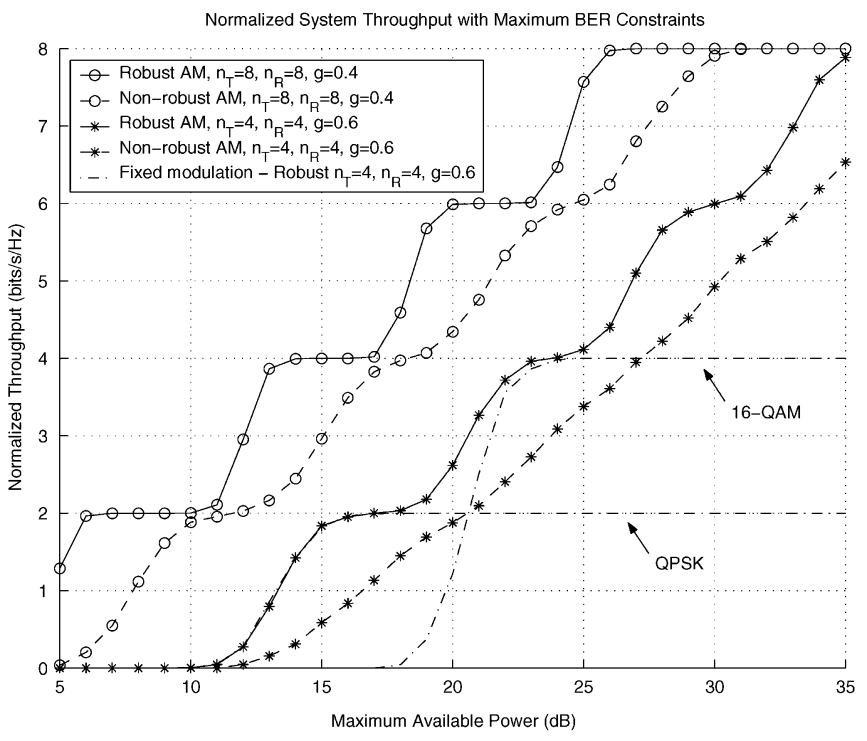

Fig. 10. Average throughput (i.e., average number of transmitted bits per channel use) for the robust and the nonrobust approaches combined with adaptive modulation taking a maximum allowed $\mathrm{BER}_{0}=10^{-3}$. The throughput has been normalized with respect to the code rate of the employed OSTBC.

considered to be spherical with a radius equal to $\sqrt{\epsilon}=g\|\hat{\mathbf{H}}\|_{F}$. This throughput has been normalized with respect to the code rate $R / T$ so that the gains provided by the robust technique itself for different number of transmit antennas can be compared directly. The techniques that are compared are the robust approach and the nonrobust classical beamforming solution. For both techniques, the mean throughput is shown as a function of the maximum available power at the transmitter. Besides, the plots regarding the application of fixed modulation formats corresponding to QPSK and 16-QAM are also given. From the figure it is concluded that, thanks to the use of the robust maximin design, very important savings in terms of transmit power can be obtained when compared to the nonrobust solution, specially when the size of the uncertainty region is high, as expected. In the same figure, the improvement of the system can also be observed when the number of antennas increases.

\section{CONCLUSION}

In this paper, a robust design strategy has been proposed for a wireless multi-antenna MIMO channel. The transmitter is designed based on a channel estimate that may have errors from different origins, such as the Gaussian noise from the estimation process, or the errors from the quantization of the channel estimate, among others. These errors have been taken into account explicitly in the design, obtaining a robust solution less sensitive to these errors. In particular, a maximin philosophy has been adopted to include robustness. This approach is characterized by attaining the best worst case performance, in terms of SNR, for any channel response in an uncertainty region centered at the channel estimate. This uncertainty region models the error in the estimate, whose shape and size have to be chosen according to the origin and amount of imperfection in the estimate.

The transmitter architecture that has been proposed is based on the concatenation of an OSTBC block, a power allocation, 
and a set of beamformers, each one connected to one of the outputs of the OSTBC and corresponding to an eigenmode of the MIMO channel estimate. The robustness has been included according to an adequate power distribution of the total transmit power among the estimated channel eigenmodes. Thanks to this optimum robust power allocation, the necessary transmit power is minimized while guaranteeing a minimum instantaneous SNR for any possible estimation error in the uncertainty region.

The mathematical optimization problem corresponding to the maximin robust power allocation has been transformed into a simple convex optimization problem that can be solved efficiently by existing software packages. For many uncertainty regions, the convex problem is quadratic and, for the case of a spherical uncertainty region, a closed-from solution exists.

Finally, this robust solution has been compared with a pure OSTBC strategy and also with the classical nonrobust beamforming strategy, corresponding to the use of only the maximum estimated channel eigenvector. From the simulations results, it has been observed that the gains in terms of saving in transmit power are quite important when compared to the nonrobust and the pure OSTBC techniques, especially when the estimation and the quantization SNR are low.

\section{APPENDIX A}

\section{Proof of Proposition 1 BASED ON THE KKT CONDITIONS}

In this appendix, an alternative proof of Proposition 1 is provided using the KKT conditions. First, some mathematical preliminaries on saddle points and concave-convex functions are given and, afterwards, the proof is provided.

\section{A. Mathematical Preliminaries}

In this subsection, the concept of saddle point is defined and two basic results are then given, since they will be useful in the following.

Definition 1: A point $\left(\mathbf{x}^{\star}, \mathbf{y}^{\star}\right) \in \mathcal{X} \times \mathcal{Y}$ is a saddle point of the function $f: \mathcal{X} \times \mathcal{Y} \rightarrow \mathbb{R}$ with respect to maximizing over $\mathcal{X}$ and minimizing over $\mathcal{Y}$ if

$$
f\left(\mathbf{x}, \mathbf{y}^{\star}\right) \leq f\left(\mathbf{x}^{\star}, \mathbf{y}^{\star}\right) \leq f\left(\mathbf{x}^{\star}, \mathbf{y}\right), \quad \forall \mathbf{x} \in \mathcal{X}, \forall \mathbf{y} \in \mathcal{Y} .
$$

Given a saddle point $\left(\mathbf{x}^{\star}, \mathbf{y}^{\star}\right)$, the saddle value is defined as $f^{\star} \triangleq f\left(\mathbf{x}^{\star}, \mathbf{y}^{\star}\right)$.

Lemma 1 [53, Corollary 37.6.2]: Let $\mathcal{X}$ and $\mathcal{Y}$ be nonempty closed bounded (compact) convex sets and let $f$ be a real continuous finite concave-convex function on $\mathcal{X} \times \mathcal{Y} .{ }^{4}$ Then, $f$ has a saddle point with respect to $\mathcal{X} \times \mathcal{Y}$.

Lemma 2 [53, Lemma 36.2]: Let $f$ be any function from a nonempty product set $\mathcal{X} \times \mathcal{Y}$ to $[-\infty,+\infty]$. If a saddle point $\left(\mathbf{x}^{\star}, \mathbf{y}^{\star}\right) \in \mathcal{X} \times \mathcal{Y}$ of $f$ exists (with respect to maximizing over $\mathcal{X}$ and minimizing over $\mathcal{Y})$, then

$$
f\left(\mathbf{x}^{\star}, \mathbf{y}^{\star}\right)=\sup _{\mathbf{x} \in \mathcal{X}} \inf _{\mathbf{y} \in \mathcal{Y}} f(\mathbf{x}, \mathbf{y})=\inf _{\mathbf{y} \in \mathcal{Y}} \sup _{\mathbf{x} \in \mathcal{X}} f(\mathbf{x}, \mathbf{y})
$$

and both $\sup _{\mathbf{x} \in \mathcal{X}} \inf _{\mathbf{y} \in \mathcal{Y}} f(\mathbf{x}, \mathbf{y})$ and $\inf _{\mathbf{y} \in \mathcal{Y}} \sup _{\mathbf{x} \in \mathcal{X}} f(\mathbf{x}, \mathbf{y})$ are attained at $\left(\mathbf{x}^{\star}, \mathbf{y}^{\star}\right)$. In other words, if a saddle point exists, the order of the supremum and infimum operators can be interchanged.

\footnotetext{
${ }^{4}$ The function $f: \mathcal{X} \times \mathcal{Y} \rightarrow \mathbb{R}$ is concave-convex if $f(\mathbf{x}, \mathbf{y})$ is concave with respect to $\mathbf{x}$ for any $\mathbf{y} \in \mathcal{Y}$, and is convex with respect to $\mathbf{y}$ for any $\mathbf{x} \in \mathcal{X}$.
}

\section{B. Proof of Proposition 1}

The function $f(\mathbf{p}, \boldsymbol{\Delta})(5)$, which is concave-convex, and the optimization sets satisfy the conditions required by Lemma 1 . Consequently, there exists a saddle point (see Definition 1) of the maximin problem (6), i.e., there exist $\mathbf{p}^{\star}$ and $\boldsymbol{\Delta}^{\star}$ fulfilling the constraints and satisfying

$$
f\left(\mathbf{p}, \boldsymbol{\Delta}^{\star}\right) \leq f\left(\mathbf{p}^{\star}, \boldsymbol{\Delta}^{\star}\right) \leq f\left(\mathbf{p}^{\star}, \boldsymbol{\Delta}\right)
$$

for any feasible $\mathrm{p}$ and $\boldsymbol{\Delta}$. The solution to the original problem (6) is $\mathbf{p}^{\star}$ and the saddle value $f^{\star} \triangleq f\left(\mathbf{p}^{\star}, \boldsymbol{\Delta}^{\star}\right)$ is $\tilde{f}\left(\mathbf{p}^{\star}\right)$ (see Lemma 2). The existence of the saddle point permits to interchange the outer and inner optimizations in the original maximin problem, obtaining the minimax problem

$$
\begin{array}{cl}
\underset{\boldsymbol{\Delta}}{\operatorname{minimize}} & \sup _{\mathbf{1}^{T} \mathbf{p} \leq P_{0}, p_{i} \geq 0} f(\mathbf{p}, \boldsymbol{\Delta}) \\
\text { subject to } & \boldsymbol{\Delta} \in \mathcal{R}
\end{array}
$$

with the advantage that the inner maximization is a linear program with linear constraints, as follows:

$$
\begin{array}{ll}
\underset{\mathbf{p}}{\operatorname{maximize}} & \sum_{i=1}^{n_{T}} p_{i}\left[\hat{\mathbf{U}}^{H}(\hat{\mathbf{H}}+\boldsymbol{\Delta})^{H}(\hat{\mathbf{H}}+\boldsymbol{\Delta}) \hat{\mathbf{U}}\right]_{i i} \\
\text { subject to } & \mathbf{1}^{T} \mathbf{p} \leq P_{0} \\
& p_{i} \geq 0, \quad \forall i .
\end{array}
$$

It can be shown that the optimum value of this maximization is the maximum element of the diagonal of the matrix $\hat{\mathbf{U}}^{H}(\hat{\mathbf{H}}+$ $\boldsymbol{\Delta})^{H}(\hat{\mathbf{H}}+\boldsymbol{\Delta}) \hat{\mathbf{U}}$ multiplied by the power budget $P_{0}$, i.e.,

$$
\begin{aligned}
\sup _{\mathbf{p} \leq P_{0}, p_{i} \geq 0} f(\mathbf{p}, \boldsymbol{\Delta}) & =P_{0} \max _{i}\left[\hat{\mathbf{U}}^{H}(\hat{\mathbf{H}}+\boldsymbol{\Delta})^{H}(\hat{\mathbf{H}}+\boldsymbol{\Delta}) \hat{\mathbf{U}}\right]_{i i} \\
& =P_{0} \max _{i}\left\{\hat{\mathbf{u}}_{i}^{H}(\hat{\mathbf{H}}+\boldsymbol{\Delta})^{H}(\hat{\mathbf{H}}+\boldsymbol{\Delta}) \hat{\mathbf{u}}_{i}\right\}
\end{aligned}
$$

where the power allocation $\mathbf{p}$ achieving this optimum value is not unique if the maximum value is attained by more than one element of the diagonal of the matrix $\hat{\mathbf{U}}^{H}(\hat{\mathbf{H}}+\boldsymbol{\Delta})^{H}(\hat{\mathbf{H}}+\boldsymbol{\Delta}) \hat{\mathbf{U}}$. As a consequence of this result, the original problem (6) can be written as the convex problem (8) shown in Proposition 1, where the dummy variable $t$ has been introduced.

Solving the convex problem (8) gives the saddle value $t^{\star}=$ $f^{\star}=f\left(\mathbf{p}^{\star}, \boldsymbol{\Delta}^{\star}\right)$ and the worst case error $\boldsymbol{\Delta}^{\star}$ of the saddle point of the problem (see Lemma 2); however, the optimal robust power distribution $\mathbf{p}^{\star}$ is still unknown. It turns out that the optimum Lagrange multipliers $\gamma_{i}^{\star}$ associated to the inequality constraints $t \geq P_{0} \hat{\mathbf{u}}_{i}^{H}(\hat{\mathbf{H}}+\boldsymbol{\Delta})^{H}(\hat{\mathbf{H}}+\boldsymbol{\Delta}) \hat{\mathbf{u}}_{i}$ in problem (8) provide the optimum normalized power distribution, i.e., $p_{i}^{\star}=P_{0} \gamma_{i}^{\star}$, as proved below.

The problem (8) can be solved by formulating the necessary and sufficient KKT conditions (Slater's condition holds since $\mathcal{R}$ has a nonempty interior), ${ }_{5}^{5}$ which, according to Lemma 2 , are satisfied by the worst case error $\Delta^{\star}$ along with the optimum dual variables. On the other hand, it is clear that $\Delta^{\star}$ is also the solution to the convex problem $\min _{\boldsymbol{\Delta}} f\left(\mathbf{p}^{\star}, \boldsymbol{\Delta}\right)$ (from the second inequality in (32)), where $\mathbf{p}^{\star}$ is the robust power distribution, and, therefore, the worst case error $\boldsymbol{\Delta}^{\star}$ must satisfy the KKT conditions for the problem $\min _{\boldsymbol{\Delta}} f\left(\mathbf{p}^{\star}, \boldsymbol{\Delta}\right)$ as well. By a simple comparison between both sets of KKT conditions, it can be seen

${ }^{5}$ The KKT conditions are necessary and sufficient conditions for optimality in a convex optimization problem if Slater's condition holds (see [8]). 
that, for $p_{i}^{\star}=P_{0} \gamma_{i}^{\star}$, the worst case error $\Delta^{\star}$ satisfies both sets of conditions and, hence, that is an optimal power allocation.

The Lagrangian of the problem (8) (characterizing for convenience and without loss of generality the uncertainty convex region $\mathcal{R}$ as the intersection of a set of convex constraints of the form $\left.f_{i}(\boldsymbol{\Delta}) \leq 0\right)^{6}$ is

$$
\begin{aligned}
L_{1}( & t, \boldsymbol{\Delta} ; \boldsymbol{\gamma}, \boldsymbol{\mu}) \\
= & t+\sum_{i=1}^{n_{T}} \gamma_{i}\left(P_{0} \hat{\mathbf{u}}_{i}^{H}(\hat{\mathbf{H}}+\boldsymbol{\Delta})^{H}(\hat{\mathbf{H}}+\boldsymbol{\Delta}) \hat{\mathbf{u}}_{i}-t\right) \\
& +\sum_{i} \mu_{i} f_{i}(\boldsymbol{\Delta}) \\
= & t\left(1-\sum_{i=1}^{n_{T}} \gamma_{i}\right) \\
& +P_{0} \operatorname{Tr}\left((\hat{\mathbf{H}}+\boldsymbol{\Delta})^{H}(\hat{\mathbf{H}}+\boldsymbol{\Delta}) \hat{\mathbf{U}} \operatorname{diag}\left(\left\{\gamma_{i}\right\}\right) \hat{\mathbf{U}}^{H}\right) \\
& +\sum_{i} \mu_{i} f_{i}(\boldsymbol{\Delta})
\end{aligned}
$$

where the equality $\sum_{i=1}^{n_{T}} \gamma_{i} \hat{\mathbf{u}}_{i} \hat{\mathbf{u}}_{i}^{H}=\hat{\mathbf{U}} \operatorname{diag}\left(\left\{\gamma_{i}\right\}\right) \hat{\mathbf{U}}^{H}$ has been used. Therefore, the KKT conditions for this problem are

$$
\begin{array}{r}
f_{i}\left(\boldsymbol{\Delta}^{\star}\right) \leq 0, \quad t^{\star} \geq P_{0} \hat{\mathbf{u}}_{i}^{H}\left(\hat{\mathbf{H}}+\boldsymbol{\Delta}^{\star}\right)^{H}\left(\hat{\mathbf{H}}+\boldsymbol{\Delta}^{\star}\right) \hat{\mathbf{u}}_{i} \\
\mu_{i}^{\star} \geq 0, \quad \gamma_{i}^{\star} \geq 0 \\
\sum_{i=1}^{n_{T}} \gamma_{i}^{\star}=1 \\
P_{0}\left(\hat{\mathbf{H}}+\boldsymbol{\Delta}^{\star}\right) \hat{\mathbf{U}} \operatorname{diag}\left(\left\{\gamma_{i}^{\star}\right\}\right) \hat{\mathbf{U}}^{H}+\sum_{i} \mu_{i}^{\star} \nabla f_{i}\left(\boldsymbol{\Delta}^{\star}\right)=\mathbf{0} \\
\mu_{i}^{\star} f_{i}\left(\boldsymbol{\Delta}^{\star}\right)=0 \\
\gamma_{i}^{\star}\left(P_{0} \hat{\mathbf{u}}_{i}^{H}\left(\hat{\mathbf{H}}+\boldsymbol{\Delta}^{\star}\right)^{H}\left(\hat{\mathbf{H}}+\boldsymbol{\Delta}^{\star}\right) \hat{\mathbf{u}}_{i}-t^{\star}\right)=0 .
\end{array}
$$

Now the Lagrangian for the problem $\min _{\Delta} f\left(\mathbf{p}^{\star}, \boldsymbol{\Delta}\right)$ is

$$
\begin{array}{r}
L_{2}(\boldsymbol{\Delta} ; \boldsymbol{\alpha})=\operatorname{Tr}\left(\hat{\mathbf{U}}^{H}(\hat{\mathbf{H}}+\boldsymbol{\Delta})^{H}(\hat{\mathbf{H}}+\boldsymbol{\Delta}) \hat{\mathbf{U}} \operatorname{diag}\left(\mathbf{p}^{\star}\right)\right) \\
+\sum_{i} \alpha_{i} f_{i}(\boldsymbol{\Delta})
\end{array}
$$

and the KKT conditions for the optimal error and multipliers are

$$
\begin{aligned}
f_{i}\left(\boldsymbol{\Delta}^{\star}\right) & \leq 0 \\
\alpha_{i}^{\star} & \geq 0 \\
\left(\hat{\mathbf{H}}+\boldsymbol{\Delta}^{\star}\right) \hat{\mathbf{U}} \operatorname{diag}\left(\mathbf{p}^{\star}\right) \hat{\mathbf{U}}^{H}+\sum_{i} \alpha_{i}^{\star} \nabla f_{i}\left(\boldsymbol{\Delta}^{\star}\right) & =\mathbf{0} \\
\alpha_{i}^{\star} f_{i}\left(\boldsymbol{\Delta}^{\star}\right) & =0 .
\end{aligned}
$$

From the comparison of both sets of KKT conditions (37)-(40) and (42)-(45), it is clear that they are satisfied by the same worst case error $\boldsymbol{\Delta}^{\star}$ taking $\alpha_{i}^{\star}=\mu_{i}^{\star}$ and $p_{i}^{\star}=P_{0} \gamma_{i}^{\star}$. Besides, from (40) it is concluded that the saddle value is $f^{\star}=$ $t^{\star}=\sum_{i=1}^{n_{t}} P_{0} \gamma_{i}^{\star} \hat{\mathbf{u}}_{i}^{H}\left(\hat{\mathbf{H}}+\boldsymbol{\Delta}^{\star}\right)^{H}\left(\hat{\mathbf{H}}+\boldsymbol{\Delta}^{\star}\right) \hat{\mathbf{u}}_{i}=f\left(\mathbf{p}^{\star}, \boldsymbol{\Delta}^{\star}\right)$. In other words, given a solution to (37)-(40), a solution to (42)-(45) is automatically obtained, which means that $\boldsymbol{\Delta}^{\star}$ is the worst case error for $\mathbf{p}^{\star}$; moreover, the pair $\left(\mathbf{p}^{\star}, \Delta^{\star}\right)$ is a saddle point of the problem and, consequently, $\mathbf{p}^{\star}$ is a robust

\footnotetext{
${ }^{6} \mathrm{An}$ arbitrary intersection of convex sets is also a convex set. In addition, the sublevel sets $\mathcal{S}_{\alpha} f$ of a convex function $f$, defined as $\mathcal{S}_{\alpha} f=\{\mathbf{x}: f(\mathbf{x}) \leq \alpha\}$, are also convex.
}

power allocation. Note that the transmit power constraint is fulfilled, since the optimum dual variables $\left\{\gamma_{i}^{\star}\right\}$ are required to satisfy $\gamma_{i}^{\star} \geq 0\left(\right.$ see (38)) and $\sum_{i=1}^{n_{T}} \gamma_{i}^{\star}=1$ (see (39)).

\section{APPENDIX B \\ PROOF OF PROPOSITION 2}

This proof is based on the proof of Proposition 1, which uses the KKT conditions, shown in Appendix A. The concave-convex function $f(\mathbf{p}, \boldsymbol{\Delta})$ in (5) can be rewritten in terms of the matrices $\overline{\mathbf{H}}$ and $\overline{\boldsymbol{\Delta}}$, which are obtained by performing a linear transformation of the original channel estimate and error matrices using the unitary matrix of estimated eigenvectors:

$$
\overline{\mathbf{H}}=\hat{\mathbf{H}} \hat{\mathbf{U}}, \quad \bar{\Delta}=\Delta \hat{\mathbf{U}}
$$

and, therefore

$$
\begin{aligned}
f(\mathbf{p}, \overline{\boldsymbol{\Delta}}) & =\operatorname{Tr}\left(\hat{\mathbf{U}}^{H}(\hat{\mathbf{H}}+\boldsymbol{\Delta})^{H}(\hat{\mathbf{H}}+\boldsymbol{\Delta}) \hat{\mathbf{U}} \operatorname{diag}(\mathbf{p})\right) \\
& =\operatorname{Tr}\left((\overline{\mathbf{H}}+\overline{\boldsymbol{\Delta}})^{H}(\overline{\mathbf{H}}+\overline{\boldsymbol{\Delta}}) \operatorname{diag}(\mathbf{p})\right) .
\end{aligned}
$$

Note that the uncertainty region defined for $\boldsymbol{\Delta}$ as $\|\boldsymbol{\Delta}\|_{F}^{2} \leq \epsilon$ can be equivalently written in terms of the transformed error $\bar{\Delta}$ as $\bar{R}=\left\{\bar{\Delta}:\|\bar{\Delta}\|_{F}^{2} \leq \epsilon\right\}$, since the multiplication by the unitary matrix $\mathbf{U}$ does not modify the value of the norm. Besides, the norms of the columns of the transformed channel matrix $\overline{\mathbf{H}}$ are related to the estimated eigenvalues by $\left\|\overline{\mathbf{h}}_{i}\right\|=$ $\sqrt{\hat{\lambda}_{i}}$.

The original maximin problem (6) can be rewritten as a minimax problem (the order of the inner and outer optimizations can be interchanged according to Lemma 2 and as used in the proof of Proposition 1, since a saddle point of the problem exists) that can be formulated as

$$
\begin{array}{ll}
\underset{\overline{\boldsymbol{\Delta}}}{\operatorname{minimize}} & \max _{i} P_{0}\left[(\overline{\mathbf{H}}+\overline{\boldsymbol{\Delta}})^{H}(\overline{\mathbf{H}}+\overline{\boldsymbol{\Delta}})\right]_{i i} \\
\text { subject to } & \|\overline{\boldsymbol{\Delta}}\|_{F}^{2} \leq \epsilon .
\end{array}
$$

The elements of the diagonal can be written as $\left[(\overline{\mathbf{H}}+\overline{\boldsymbol{\Delta}})^{H}(\overline{\mathbf{H}}+\right.$ $\overline{\boldsymbol{\Delta}})]_{i i}=\left\|\overline{\mathbf{h}}_{i}+\overline{\boldsymbol{\delta}}_{i}\right\|^{2}$. Consider now the minimization with respect to each $\overline{\boldsymbol{\delta}}_{i}$, i.e., to each column of the matrix $\overline{\boldsymbol{\Delta}}$. The vector $\overline{\boldsymbol{\delta}}_{i}$ with norm $\left\|\overline{\boldsymbol{\delta}}_{i}\right\|=c_{i}$ that minimizes $\left\|\overline{\mathbf{h}}_{i}+\overline{\boldsymbol{\delta}}_{i}\right\|^{2}$ is $\overline{\boldsymbol{\delta}}_{i}^{\star}=-c_{i} \overline{\mathbf{h}}_{i} /\left\|\overline{\mathbf{h}}_{i}\right\|$. Using this result, the minimized $i$ th component of the diagonal can be written as

$$
\begin{aligned}
& {\left[\left(\overline{\mathbf{H}}+\bar{\Delta}^{\star}\right)^{H}\left(\overline{\mathbf{H}}+\bar{\Delta}^{\star}\right)\right]_{i i}} \\
& \quad=\left\|\overline{\mathbf{h}}_{i}\left(1-\frac{1}{\left\|\overline{\mathbf{h}}_{i}\right\|} c_{i}\right)\right\|^{2} \\
& \quad=\left\|\overline{\mathbf{h}}_{i}\right\|^{2}\left(1-\frac{1}{\left\|\overline{\mathbf{h}}_{i}\right\|} c_{i}\right)^{2}=\left(\left\|\overline{\mathbf{h}}_{i}\right\|-c_{i}\right)^{2} .
\end{aligned}
$$

According to this, the problem (48) can be equivalently expressed as

$$
\begin{array}{cl}
\underset{t, \mathbf{c}}{\operatorname{minimize}} & t \\
\text { subject to } & t \geq P_{0}\left(\left\|\overline{\mathbf{h}}_{i}\right\|-c_{i}\right)^{2}, \quad \forall i \\
& \sum_{i=1}^{n_{T}} c_{i}^{2}=\|\mathbf{c}\|^{2} \leq \epsilon
\end{array}
$$

where $\mathbf{c}=\left[c_{1} \cdots c_{n_{T}}\right]^{T}$.

It is now clear how to find the optimum solution to this problem according to the points below, taking into account that 


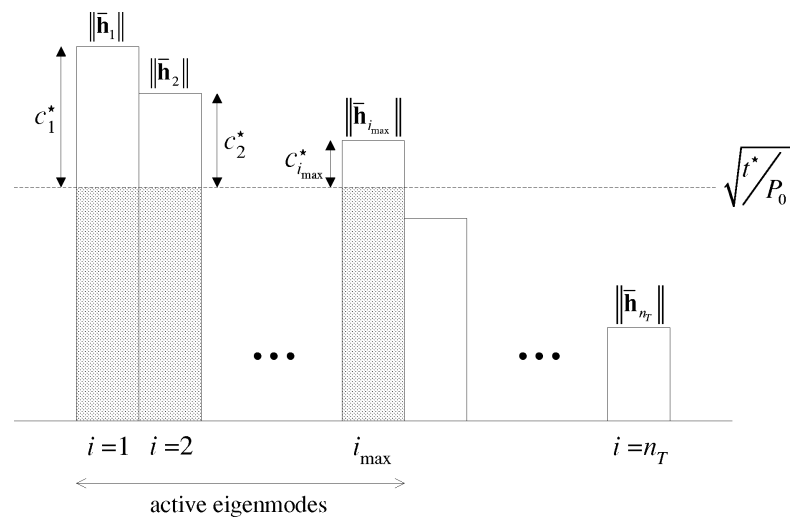

Fig. 11. Representation of the optimal solution for the case of a spherical uncertainty region.

the eigenvalues $\left\{\hat{\lambda}_{i}\right\}$ are sorted in decreasing order and that $\left\|\overline{\mathbf{h}}_{i}\right\|=\sqrt{\hat{\lambda}_{i}}$.

1) Detection of saddle value equal to 0 : In case that $\sum_{i=1}^{n_{T}}\left\|\overline{\mathbf{h}}_{i}\right\|^{2}=\|\overline{\mathbf{H}}\|_{F}^{2}=\|\hat{\mathbf{H}}\|_{F}^{2} \leq \epsilon$, then the worst-error is attained when $c_{i}^{\star}=\left\|\overline{\mathbf{h}}_{i}\right\|$, which corresponds to $\Delta^{\star}=-\hat{\mathbf{H}}$, and the saddle value $t^{\star}$ is 0 , which means that no SNR can be guaranteed for any power allocation.

2) Detection of the number of active eigenmodes: When the saddle value is different from 0 , some of the inequality constraints in (50) are fulfilled with equality, whereas the value of $c_{i}$ for the other constraints is 0 . This is the optimum solution, since, in case that there exists an index $j$ such that $c_{j}>0$ but the corresponding constraint is not fulfilled with equality (i.e., $\left.t>P_{0}\left(\left\|\overline{\mathbf{h}}_{j}\right\|-c_{j}\right)^{2}\right)$, then the value of $t$ can be reduced by decreasing the value of $c_{j}$ and increasing $c_{i}, \forall i \neq j$, while still fulfilling $\|\mathbf{c}\|^{2} \leq \epsilon$. Consequently, the optimum solution is attained when $\left\|\overline{\mathbf{h}}_{i}\right\|-c_{i}$ is constant for the active constraints and the value of $t$ cannot be further reduced since $\|\mathbf{c}\|^{2}=\epsilon$ (see Fig. 11 for an example of an optimal solution). Taking all this into account, the number of active constraints is the maximum index $i_{\max }$ such that the following inequality is fulfilled:

$$
\sum_{i=1}^{i_{\max }}\left(\left\|\overline{\mathbf{h}}_{i}\right\|-\left\|\overline{\mathbf{h}}_{i_{\max }}\right\|\right)^{2}<\epsilon .
$$

Once this index has been calculated by a simple finite iteration, the optimum values of the coefficients $\left\{c_{i}\right\}$ for the active constraints can be expressed as a function of the constant for the last active constraint $c_{i_{\max }}$ (note that for the other constraints, the optimum constants are equal to 0 , i.e., $c_{i}^{\star}=0, i>i_{\max }$ ), as follows:

$$
\begin{aligned}
& \left\|\overline{\mathbf{h}}_{i}\right\|-c_{i}^{\star}=\left\|\overline{\mathbf{h}}_{i_{\max }}\right\|-c_{i_{\max }^{\star}}^{\star} \\
& \quad \Rightarrow c_{i}^{\star}=\left\|\overline{\mathbf{h}}_{i}\right\|-\left\|\overline{\mathbf{h}}_{i_{\max }}\right\|+c_{i_{\max }}^{\star}, \quad i=1, \ldots, i_{\max } .
\end{aligned}
$$

Using this result, $c_{i_{\max }}^{\star}$ can be easily calculated by taking the positive solution to the following second degree equation resulting from the constraint $\sum_{i=1}^{n_{T}} c_{i}^{2}=\epsilon$ :

$$
\begin{aligned}
& \sum_{i=1}^{n_{T}} c_{i}^{\star 2}=\sum_{i=1}^{i_{\max }}\left(\left\|\overline{\mathbf{h}}_{i}\right\|-\left\|\overline{\mathbf{h}}_{i_{\max }}\right\|+c_{i_{\max }}^{\star}\right)^{2}=\epsilon \\
& \Rightarrow\left(c_{i_{\max }^{\star}}^{{ }^{2}}\right)^{2} i_{\max }+2 \sum_{i=1}^{i_{\max }}\left(\left\|\overline{\mathbf{h}}_{i}\right\|-\left\|\overline{\mathbf{h}}_{i_{\max }}\right\|\right) c_{i_{\max }}^{\star} \\
& \quad+\sum_{i=1}^{i_{\max }}\left(\left\|\overline{\mathbf{h}}_{i}\right\|-\left\|\overline{\mathbf{h}}_{i_{\max }}\right\|\right)^{2}-\epsilon=0 .
\end{aligned}
$$

Collecting all these results, the worst case error $\Delta^{\star}$ can be finally calculated as

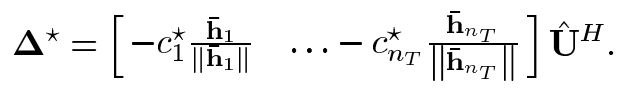

The optimal robust power allocation can be obtained as the power $\mathbf{p}^{\star}$ such that the worst case error is a solution to $\min _{\boldsymbol{\Delta}} f\left(\mathbf{p}^{\star}, \boldsymbol{\Delta}\right)$, i.e., $\boldsymbol{\Delta}^{\star}$ satisfies the corresponding KKT conditions. The Lagrangian associated to the problem is

$$
\begin{array}{r}
L(\boldsymbol{\Delta} ; \mu)=\operatorname{Tr}\left(\hat{\mathbf{U}}^{H}(\hat{\mathbf{H}}+\boldsymbol{\Delta})^{H}(\hat{\mathbf{H}}+\boldsymbol{\Delta}) \hat{\mathbf{U}} \operatorname{diag}\left(\mathbf{p}^{\star}\right)\right) \\
+\mu\left(\operatorname{Tr}\left(\boldsymbol{\Delta}^{H} \boldsymbol{\Delta}\right)-\epsilon\right)
\end{array}
$$

and, therefore, one of the KKT conditions is

$$
\begin{aligned}
\left(\hat{\mathbf{H}}+\boldsymbol{\Delta}^{\star}\right) & \hat{\mathbf{U}} \operatorname{diag}\left(\mathbf{p}^{\star}\right) \hat{\mathbf{U}}^{H}+\mu^{\star} \boldsymbol{\Delta}^{\star}=\mathbf{0} \\
\Rightarrow & \left(\hat{\mathbf{H}}+\boldsymbol{\Delta}^{\star}\right) \hat{\mathbf{U}} \operatorname{diag}\left(\mathbf{p}^{\star}\right)+\mu^{\star} \boldsymbol{\Delta}^{\star} \hat{\mathbf{U}}=\mathbf{0} \\
& \left(\overline{\mathbf{H}}+\overline{\boldsymbol{\Delta}}^{\star}\right) \operatorname{diag}\left(\mathbf{p}^{\star}\right)+\mu^{\star} \overline{\boldsymbol{\Delta}}^{\star}=\mathbf{0}
\end{aligned}
$$

which has to be satisfied at the worst case error $\Delta^{\star}$ and for the robust power allocation $\left\{p_{i}^{\star}\right\}$. From this equation, the power to be allocated to the $i$ th estimated eigenmode can be calculated as

$$
\begin{aligned}
& \left(\overline{\mathbf{h}}_{i}+\overline{\boldsymbol{\delta}}_{i}^{\star}\right) p_{i}^{\star}+\mu^{\star} \overline{\boldsymbol{\delta}}_{i}^{\star}=\mathbf{0} \\
& \Rightarrow \overline{\mathbf{h}}_{i}\left(1-\frac{c_{i}^{\star}}{\left\|\overline{\mathbf{h}}_{i}\right\|}\right) p_{i}^{\star}-\mu^{\star} \overline{\mathbf{h}}_{i} \frac{c_{i}^{\star}}{\left\|\overline{\mathbf{h}}_{i}\right\|}=\mathbf{0} \\
& \Rightarrow p_{i}^{\star}=\mu^{\star} \frac{\frac{c_{i}^{\star}}{\left\|\hat{\mathbf{h}}_{i}\right\|}}{1-\frac{c_{i}^{\star}}{\left\|\overline{\mathbf{h}}_{i}\right\|}}=\mu^{\star} \frac{c_{i}^{\star}}{\sqrt{\hat{\lambda}_{i}}-c_{i}^{\star}} \\
& = \begin{cases}\mu^{\star} \frac{\sqrt{\hat{\lambda}_{i}}-\sqrt{\hat{\lambda}_{\text {max }}}+c_{i_{\max }}^{\star}}{\sqrt{\hat{\lambda}_{i_{\max }}}-c_{i_{\max }}}, & 1 \leq i \leq i_{\max } \\
0, & i>i_{\max }\end{cases}
\end{aligned}
$$

where $\mu^{\star}$ is a normalization factor such that $\sum_{i=1}^{n_{T}} p_{i}^{\star}=P_{0}$. By defining the constant $\alpha=\sqrt{\hat{\lambda}_{i_{\max }}}-c_{i_{\max }}^{\star}$, absorbing the factor $1 / \alpha$ in $\mu^{\star}$, and introducing the change of variable $c_{i_{\max }}^{\star}=$ $\sqrt{\hat{\lambda}_{i_{\max }}}-\alpha$ in (54), the results in Proposition 2 are directly obtained, including (28). 


\section{REFERENCES}

[1] S. M. Alamouti, "A simple transmit diversity technique for wireless communications," IEEE J. Sel. Areas Commun., vol. 16, no. 8, pp. 1451-1458, Oct. 1998

[2] G. J. Foschini, "Layered space-time architecture for wireless communication in a fading environment when using multi-element antennas," Bell Labs. Tech. J., vol. 1, no. 2, pp. 41-59, Autumn 1996.

[3] V. Tarokh, N. Seshadri, and A. R. Calderbank, "Space-time codes for high data rate wireless communications: Performance criterion and code construction," IEEE Trans. Inf. Theory, vol. 44, no. 2, pp. 744-765, Mar. 1998.

[4] V. Tarokh, H. Jafharkani, and A. R. Calderbank, "Space-time block codes from orthogonal designs," IEEE Trans. Inf. Theory, vol. 45, no. 5, pp. 1456-1467, Jul. 1999.

[5] G. Ganesan and P. Stoica, "Space-time block codes: A maximum SNR approach," IEEE Trans. Inf. Theory, vol. 47, no. 4, pp. 1650-1656, May 2001

[6] D. P. Palomar, J. M. Cioffi, and M. A. Lagunas, "Joint Tx-Rx beamforming design for multicarrier MIMO channels: A unified framework for convex optimization,” IEEE Trans. Signal Process., vol. 51, no. 9, pp. 2381-2401, Sep. 2003

[7] D. P. Palomar, M. A. Lagunas, and J. M. Cioffi, "Optimum linear joint transmit-receive processing for MIMO channels with QoS constraints," IEEE Trans. Signal Process., vol. 52, no. 5, pp. 1179-1197, May 2004.

[8] S. Boyd and L. Vandenberghe, Convex Optimization. Cambridge, U.K.: Cambridge Univ. Press, 2004.

[9] K. Zhou, J. C. Doyle, and K. Glover, Robust and Optimal Control. Upper Saddle River, NJ: Prentice-Hall International, 1996.

[10] B. Hassibi, A. H. Sayed, and T. Kailath, Indefinite Quadratic Estimation and Control: A Unified Approach to $\mathcal{H}_{2}$ and $\mathcal{H}_{\infty}$ Theories. Philadelphia, PA: SIAM, 1999.

[11] A. Pascual-Iserte, A. I. Pérez-Neira, and M. A. Lagunas, "On power allocation strategies for maximum signal to noise and interference ratio in an OFDM-MIMO system," IEEE Trans. Wireless Commun., vol. 3, no. 3, pp. 808-820, May 2004.

[12] J. Choi, "Performance analysis for transmit antenna diversity with/without channel information," IEEE Trans. Veh. Technol., vol. 51, no. 1, pp. 101-113, Jan. 2002.

[13] — - "Performance limitation of closed-loop transmit antenna diversity over fast Rayleigh fading channels," IEEE Trans. Veh. Technol., vol. 51, no. 4, pp. 771-775, Jul. 2002.

[14] S. Zhou and G. B. Giannakis, "How accurate channel prediction needs to be for transmit-beamforming with adaptive modulation over Rayleigh MIMO channels?," IEEE Trans. Wireless Commun., vol. 3, no. 4, pp. 1285-1294, Jul. 2004.

[15] M. Bengtsson and B. Ottersten, "Optimal and suboptimal transmit beamforming," in Handbook of Antennas in Wireless Communications, L. C. Godara, Ed. Boca Raton, FL: CRC, 2001

[16] D. P. Palomar, "A Unified framework for communications through MIMO channels," Ph.D. dissertation, Dept. Signal Theory and Communications, Technical Univ. Catalonia (UPC), Barcelona, Spain, May 2003.

[17] A. Narula, M. J. Lopez, M. D. Trot, and G. W. Wornell, "Efficient use of side information in multiple-antenna data transmission over fading channels," IEEE J. Sel. Areas Commun., vol. 16, no. 8, pp. 1423-1436, Oct. 1998.

[18] A. Wittneben, "Optimal predictive TX combining diversity in correlated fading for microcellular mobile radio applications," in Proc. IEEE Global Telecommunications Conf. (GLOBECOM 1995), Nov. 1995, pp. 13-17.

[19] A. Pascual-Iserte, A. I. Pérez-Neira, and M. A. Lagunas, "Exploiting transmission spatial diversity in frequency selective systems with feedback channel," in Proc. IEEE Int. Conf. Acoustics, Speech, Signal Processing (ICASSP 2003), vol. 4, Apr. 2003, pp. 85-88.

[20] G. Jöngren, M. Skoglund, and B. Ottersen, "Combining beamforming and orthogonal space-time block coding," IEEE Trans. Inf. Theory, vol. 48, no. 3, pp. 611-627, Mar. 2002.

[21] F. Rey, M. Lamarca, and G. Vázquez, "Transmit filter optimization based on partial CSI knowledge for wireless applications," in Proc. IEEE Int. Conf. Communications (ICC 2003), vol. 4, Anchorage, AK, May 11-15, 2003, pp. 2567-2571

[22] P. Xia, S. Zhou, and G. B. Giannakis, "Adaptive MIMO-OFDM based on partial channel state information," IEEE Trans. Signal Process., vol. 52, no. 1, pp. 202-213, Jan. 2004.
[23] S. Zhou and G. B. Giannakis, "Optimal transmitter eigen-beamforming and space-time block coding based on channel mean feedback," IEEE Trans. Signal Process., vol. 50, no. 10, pp. 2599-2613, Oct. 2002.

[24] — - "Optimal transmitter eigen-beamforming and space-time block coding based on channel correlations," IEEE Trans. Inf. Theory, vol. 49, no. 7, pp. 1673-1690, Jul. 2003.

[25] A. Goldsmith, S. A. Jafar, N. Jindal, and S. Vishwanath, "Capacity limits of MIMO channels," IEEE J. Sel. Areas Commun., vol. 21, no. 5, pp. 684-702, Jun. 2003

[26] S. A. Kassam and H. V. Poor, "Robust techniques for signal processing: A survey," in Proc. IEEE, vol. 73, Mar. 1985, pp. 433-481.

[27] S. Verdú and V. Poor, "On minimax robustness: A general approach and applications," IEEE Trans. Inf. Theory, vol. 30, no. 2, pp. 328-340, Mar 1984.

[28] M. Osborne and A. Rubinstein, A Course in Game Theory. Cambridge, MA: MIT Press, 1994

[29] S. A. Vorobyov, A. B. Gershman, and Z.-Q. Luo, "Robust adaptive beamforming using worst-case performance optimization: A solution to the signal mismatch problem," IEEE Trans. Signal Process., vol. 51, no. 2, pp. 313-324, Feb. 2003.

[30] S. Shahbazpanahi, A. B. Gershman, Z.-Q. Luo, and K. M. Wong, "Robust adaptive beamforming for general-rank signal models," IEEE Trans. Signal Process., vol. 51, no. 9, pp. 2257-2269, Sep. 2003.

[31] R. A. Monzingo and T. W. Miller, Introduction to Adaptive Arrays. New York: Wiley, 1980.

[32] R. Lorenz and S. P. Boyd, "Robust minimum variance beamforming," IEEE Trans. Signal Process., vol. 53, no. 5, pp. 1684-1696, May 2005.

[33] J. Li, P. Stoica, and Z. Wang, "On robust capon beamforming and diagonal loading," IEEE Trans. Signal Process., vol. 51, no. 7, pp. 1702-1715, Jul. 2003

[34] P. Stoica, Z. Wang, and J. Li, "Robust Capon beamforming," IEEE Signal Process. Lett., vol. 10, no. 6, pp. 172-175, Jun. 2003.

[35] Y. C. Eldar and N. Merhav, "A competitive minimax approach to robust estimation of random parameters," IEEE Trans. Signal Process., vol. 52 , no. 7, pp. 1931-1946, Jul. 2004

[36] Y. Rong, S. Shahbazpanahi, and A. B. Gershman, "Robust linear receivers for space-time block coded multiple-access MIMO wireless systems," in Proc. IEEE Int. Conf. Acoustics, Speech, Signal Processing (ICASSP 2004), vol. 2, May 2004, pp. 17-21.

[37] A. Abdel-Samad, A. B. Gershman, and T. N. Davidson, "Robust transmit beamforming based on imperfect channel feedback," in Proc. IEEE Vehicular Technology Conf. (VTC Fall 2004), vol. 3, Sep. 2004, pp. 2049-2053.

[38] M. Bengtsson and B. Ottersten, "Optimal downlink beamforming using semidefinite optimization," in Proc. 37th Annu. Allerton Conf. Coтmunications, Control, Computing, Sep. 1999, pp. 987-996.

[39] M. Biguesh, S. Shahbazpanahi, and A. B. Gershman, "Robust power adjustment for transmit beamforming in cellular communications systems," in Proc. IEEE Int. Conf. Acoustics, Speech, Signal Processing (ICASSP 2003), vol. 5, Apr. 2003, pp. 105-108

[40] D. P. Bertsekas, Nonlinear Programming, 2nd ed. Belmont, MA Athena Scientific, 1999

[41] S. A. Jafar and A. Goldsmith, "Transmitter optimization and optimality of beamforming for multiple antenna systems," IEEE Trans. Wireless Commun., vol. 3, no. 4, pp. 1165-1175, Jul. 2004.

[42] B. Hassibi and B. M. Hochwald, "High-rate codes that are linear in space and time," IEEE Trans. Inf. Theory, vol. 48, no. 7, pp. 1804-1824, Jul. 2002.

[43] H. Jafarkhani, "A quasi-orthogonal space-time block code," IEEE Trans. Commun., vol. 49, no. 1, pp. 1-4, Jan. 2001.

[44] A. Pascual-Iserte, A. I. Pérez-Neira, and M. A. Lagunas, "A maximin approach for robust MIMO design: Combining OSTBC and beamforming with minimum transmission power requirements," in Proc. IEEE Int Conf. Acoustics, Speech, Signal Processing (ICASSP 2004), vol. 2, May 2004, pp. 1-4.

[45] D. P. Palomar, A. Pascual-Iserte, J. M. Cioffi, and M. A. Lagunas, "Convex optimization theory applied to joint transmitter-receiver design in MIMO channels," in Space-Time Processing for MIMO Communications, A. B. Gershman and N. D. Sidiropoulos, Eds. New York: Wiley, ch. 8, pp. 269-318.

[46] R. T. Rockafellar, "Saddle-points and convex analysis," in Differential Games and Related Topics, H. W. Kuhn and G. P. Szego, Eds. Amsterdam, The Netherlands: North-Holland, 1971, pp 109-127.

[47] S. Žaković and C. Pantelides, "An interior point method algorithm for computing saddle points of constrained continuous minimax," Ann. Oper. Res., vol. 99, pp. 59-77, Dec. 2000. 
[48] J. F. Sturm, "Using sedumi 1.02, a MATLAB toolbox for optimization over symmetric cones," Optimization Meth. Softw., vol. 11-12, pp. 625-653, 1999.

[49] S. M. Kay, Fundamentals of Statistical Signal Processing: Estimation Theory. Englewood Cliffs, NJ: Prentice-Hall International, 1993.

[50] A. K. Jain, Fundamentals of Digital Signal Processing. Englewood Cliffs, NJ: Prentice-Hall International, 1989.

[51] D. J. Love, R. W. Heath, W. Santipach, and M. L. Honig, "What is the value of limited feedback for MIMO channels?," IEEE Commun. Mag., vol. 42, no. 10, pp. 54-59, Oct. 2004.

[52] S. T. Chung and A. J. Goldsmith, "Degrees of freedom in adaptive modulation: A unified view," IEEE Trans. Commun., vol. 49, no. 9, pp. 1561-1571, Sep. 2001.

[53] R. T. Rockafellar, Convex Analysis, 2nd ed. Princeton, NJ: Princeton Univ. Press, 1970.

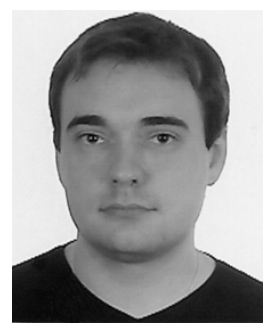

Antonio Pascual-Iserte (S'01) was born in Barcelona, Spain, in 1977. He received the Electrical Engineering degree and the Ph.D. degree (with highest honors) from the Universitat Politècnica de Catalunya (UPC), Barcelona, Spain, in 2000 and 2005 , respectively.

From September 1998 to June 1999, he worked on microprocessor programming with the Electronic Engineering Department, UPC. From June 1999 to December 2000, he was with Retevision R\&D, Barcelona, Spain, where he worked on the implantation of the DVB-T and T-DAB networks in Spain. In January 2001, he joined the Department of Signal Theory and Communications, UPC, where he worked as a Research Assistant until September 2003 under a grant from the Catalan Government. Since September 2003, he has been an Assistant Professor at UPC. He has also been a Visiting Researcher with the Telecommunications Technological Center of Catalonia (CTTC), Barcelona, Spain, since January 2002. Currently, he is involved in several national and European research projects. He has published several papers at international conferences and in journals on the topics of array signal processing and multiple-input-multiple-output (MIMO) channels.

Dr. Pascual-Iserte was awarded with the First National Prize of 2000-2001 University Education by the Spanish Ministry of Education and Science.

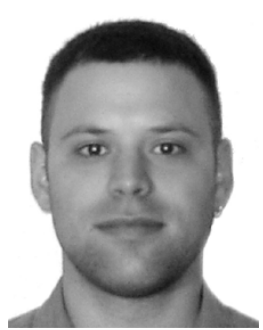

Daniel Pérez Palomar (S'99-M'03) received the Electrical Engineering degree and the Ph.D. degree (both with honors) from the Technical University of Catalonia (UPC), Barcelona, Spain, in 1998 and 2003, respectively.

In 1998, he was with the Department of Electronic Engineering, King's College London (KCL), London, U.K. From January 1999 to December 2003, he was a Research Assistant with the Department of Signal Theory and Communications, UPC. From April to November 2001, he held a visiting research appointment at the Department of Electrical Engineering, Stanford University, Stanford, CA. From January to December 2002, he was a Visiting Researcher with the Telecommunications Technological Center of Catalonia (CTTC), Barcelona, Spain. From August to November 2003, he was a Guest Researcher at the Department of Signals, Sensors, and Systems, Royal Institute of Technology (KTH), Stockholm, Sweden. From November 2003 to February 2004, he was a Visiting Researcher with the INFOCOM Department, University of Rome "La Sapienza," Rome, Italy. Since March 2004, he has been a Fulbright Research Fellow at Princeton University, Princeton, NJ. His primary research interests include information-theoretic and communication aspects of wireless multiple-input-multiple-output (MIMO) channels and array signal processing, with special emphasis on convex optimization theory applied to communication systems.

Dr. Palomar received the 2004 Young Author Best Paper Award by the IEEE Signal Processing Society; the 2002-2003 Best Ph.D. prize within the area of Information Technologies and Communications by the Technical University of Catalonia (UPC); the 2002-2003 Rosina Ribalta first prize for the Best Doctoral Thesis with the areas of Information Technologies and Communications by the Epson Foundation; and the 2004 prize for the Best Doctoral Thesis in Advanced Mobile Communications by the Vodafone Foundation and Colegio Oficial de Ingenieros de Telecomunicación (COIT).

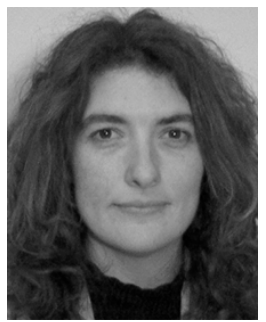

Ana I. Pérez-Neira (S'92-M'95-SM'01) was born in Zaragoza, Spain, in 1967. She received the Telecommunication Engineering and Ph.D. degrees from the Universitat Politècnica de Catalunya (UPC), Barcelona, Spain, in 1991 and 1995, respectively.

In 1991, she joined the Department of Signal Theory and Communications, UPC, where she carried out research activities in the field of higher order statistics and statistical array processing. In 1992, she became Lecturer, and since 1996, she has been an Associate Professor with UPC, where she teaches and coordinates graduate and undergraduate courses in statistical signal processing, analog and digital communications, mathematical methods for communications, and nonlinear signal processing. She is author of ten journal and more than 50 conference papers in the area of statistical signal processing and fuzzy processing, with applications to mobile/satellite communications systems. She has coordinated several private, national public, and European founded projects.

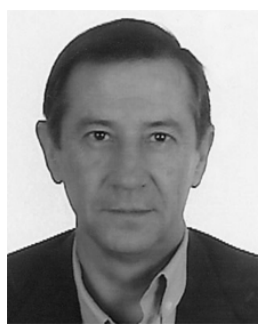

Miguel Ángel Lagunas (S'73-S'78-SM'89-F'97) was born in Madrid, Spain, in 1951. He received the Telecommunication Engineer degree from the Universitat Politènica de Madrid (UPM), Madrid, Spain, in 1973 and the Ph.D. degree in telecommunications from the Universitat Politècnica de Barcelona (UPB), Barcelona, Spain, in 1976.

From 1971 to 1973, he was a Research Assistant with the Semiconductor Lab ETSIT, Madrid, Spain. From 1973 to 1979, he was a Teacher Assistant in Network Synthesis and Semiconductor Electronics. From 1979 to 1982, he was an Associate Professor of digital signal processing, Barcelona, Spain. Since 1983, he has been a Full Professor at the Universitat Politècnica de Catalunya (UPC), Barcelona, where he teaches courses in signal processing, array processing, and digital communications. He was Project Leader of high-speed SCMA (1987-1989) and ATM (1994-1995) cable network. He is also Codirector of the first projects for the European Spatial Agency and the European Union, providing engineering demonstration models on smart antennas for satellite communications using DS and FH systems (1986) and mobile communications GSM (Tsunami, 1994). Currently, he is Director of the Telecommunications Technological Center of Catalonia (CTTC), Barcelona, Spain. His research interests include spectral estimation, adaptive systems, and array processing. His technical activities are in advanced front ends for digital communications combining spatial with frequency-time and coding diversity.

Dr. Lagunas was Vice-President for Research of UPC from 1986 to 1989 and Vice-Secretary General for Research, CICYT, Spain, from 1995 to $1996 . \mathrm{He}$ is a member-at-large of EURASIP and an Elected Member of the Academy of Engineers of Spain and of the Academy of Science and Art of Barcelona. He was a Fullbright Scholar at the University of Boulder, Boulder, CO. 